\title{
HOMOGENEOUS ALMOST COMPLEX STRUCTURES IN DIMENSION 6 WITH SEMI-SIMPLE ISOTROPY
}

\author{
D. V. ALEKSEEVSKY, B. S. KRUGLIKOV, H. WINTHER
}

\begin{abstract}
We classify invariant almost complex structures on homogeneous manifolds of dimension 6 with semi-simple isotropy. Those with non-degenerate Nijenhuis tensor have the automorphism group of dimension either 14 or 9 . An invariant almost complex structure with semisimple isotropy is necessarily either of specified 6 homogeneous types or a left-invariant structure on a Lie group. For integrable invariant almost complex structures we classify all compatible invariant Hermitian structures on these homogeneous manifolds, indicate their integrability properties (Kähler, SNK, SKT) and mark the other interesting geometric properties (including the Gray-Hervella type).
\end{abstract}

\section{INTRODUCTION AND MAIN RESULtS}

Consider an almost complex manifold $(M, J), J^{2}=\mathbf{- 1}$. If $M$ is closed, the automorphisms of $J$ form a Lie group [BKW]. In general the automorphism group can be infinite-dimensional, but finite-dimensionality can be guaranteed by additional local (non-degeneracy of the Nijenhuis tensor $\mathrm{K}_{1}$ ) or global (Kobayashi partial hyperbolicity [Ko, $[\mathrm{KO}]$ ) conditions.

An almost complex structure is integrable if the Nijenhuis tensor $N_{J}$ vanishes [NW]. In real dimension 6 (complex dimension 3) non-degeneracy of the Nijenhuis tensor means that $N_{J}: \Lambda_{\mathbb{C}}^{2} T M \rightarrow T M$ is a ( $\mathbb{C}$-antilinear) isomorphism. Such structures are important in applications to critical points of the Hitchin-type functionals and nearly Kähler geometry [Br, $\mathrm{V}]$.

As proven in $\left[\mathrm{K}_{2}\right.$ the local automorphism group $G$ of the structure $J$ on $M^{6}$ with non-degenerate $N_{J}$ has dimension at most 14 , and that this bound is achieved only for the $G_{2}$-invariant almost complex structures]: either $G_{2}^{c}$ invariant $J$ on $S^{6}$ or $G_{2}^{*}$-invariant $J$ on its non-compact version $S^{2,4}$. It is natural to ask what is the next submaximal (= maximal among structures that are not locally $G_{2}$-invariant) dimension of the automorphism group.

As one can expect this is still transitive, we confine to (locally) homogeneous structures. But their classification is cumbersome, and so we restrict further by requiring the isotropy group $H$ to be semi-simple. This generalizes the assumption of [Wo. In this reference the almost complex structures on $G / H$ with irreducible isotropy $H$ were classified. Here we extend this

\footnotetext{
${ }^{1}$ We denote the compact real form of $G_{2}$ by $G_{2}^{c} \subset S O(7)$ and the split real form with the trivial center by $G_{2}^{*} \subset S O(3,4)$.
} 
classification in dimension 6 . The obtained structures $J$ possess abundant symmetries ( $\operatorname{dim} G \geq 9$ ) by construction.

Theorem 1. The only homogeneous almost complex structures on the homogeneous space $M^{6}=G / H$ with semi-simple isotropy group $H$ are (up to a covering and a quotient by a discrete central subgroup):

(I) the homogeneous almost complex structure on $S^{6}=G_{2}^{c} / S U(3)$ or on $S^{2,4}=G_{2}^{*} / S U(1,2)$;

$\left(\mathrm{II}_{1}\right)$ 4-parametric family on $U(3) / S U(2), U(2,1) / S U(2)$;

$\left(\mathrm{II}_{2}\right)$ 4-parametric family on $U(2,1) / S U(1,1)$, and 2-parametric on $G L(3) / S U(1,1)$;

(III) left-invariant almost complex structures on a 6D Lie group.

The tables of the latter structures are given in the Appendix, see also Theorem 3. The structures of type II are described in Section 6.

Remark 1. As written above, the possible $M^{6}$ are obtained from the universal covering group $G$ by additional discrete quotient $M=\Gamma \backslash G / H$. These central subgroups $\Gamma \subset G$ can be completely described. For instance, instead of $U(3) / S U(2)$ we get $G=\mathbb{R}^{1} \times S U(3), H=S U(2)$ and $\Gamma$ is one of the 4 obvious discrete subgroups of the center $Z(G)=\mathbb{R} \times \mathbb{Z}_{3}$. Similarly, we get $G=\mathbb{R} \times \widehat{S U(2,1)}$ or $\mathbb{R} \times \widetilde{S L(3)}$ for other type II cases. However the invariant almost complex structure $J$ on $M=G / H$ depends in these cases only on 2 parameters, since the torus gets covered by a cylinder, see the details of the construction in Section 6 .

Remark 2. In dimension 4 all complex representations with semi-simple $H \subset G L(2, \mathbb{C})$ lead to the flat structure $G=H \ltimes \mathbb{C}^{2}$ and so $M^{4}=\mathbb{C}^{2}$. If we allow $H$ to be reductive, then 4 new cases appear:

- $S U(3) / U(2)=\mathbb{C} P^{2}$,

- $S U(2,1) / U(2)=B^{4}$,

- $S U(2,1) / U(1,1)=\mathbb{C} P^{2} \backslash\{p t\} \simeq \mathbb{C} P^{1} \times \mathbb{C}$

- $S L(3) / U(1,1)=\mathbb{R} P^{2} \times \mathbb{R} P^{2 \vee} \backslash \mathbb{P}\{(v, p): v \cdot p=0\} \simeq T \mathbb{R} P^{2}$.

In all these cases $J$ is integrable (complex structure).

Our method can be used to extend the classification to the reductive isotropy $H$ in dimension 6 as well, which includes such complex manifolds as $\mathbb{C} P^{3}=$ $S U(4) / U(3)$, but the tables become rather big.

We will also examine the invariant (pseudo-)Riemannian and almost symplectic structures on these homogeneous 6-manifolds $G / H$, specifying (in the case they are compatible with the almost complex structure) which of them are Hermitian, Kähler, strongly nearly Kähler (SNK), strongly Kähler with torsion (SKT) and discuss the Gray-Hervella classes of them.

The SNK condition is closely related with the condition of non-degeneracy of $N_{J}$ (recall that this means $\left.N_{J}\left(\Lambda^{2} T M\right)=T M\right)$. For the Calabi almost complex structure $J$ on $S^{6}$ it is known that its automorphism group is the compact real form $G_{2}^{c}$. Similarly the split real form $G_{2}^{*}$ is the symmetry group 
of the homogeneous structure $J$ on the pseudo-sphere $S^{2,4}$ (in both cases $\operatorname{dim} \operatorname{Aut}(M, J)=14)$. It turns out that for the other cases of Theorem 1 with non-degenerate tensor $N_{J}$ the local symmetries of $J$ (and hence the global ones) are only the obvious ones.

Theorem 2. Let $J$ be an invariant almost complex structure on the homogeneous space $M=G / H$ from Theorem 1 . Assume that the Nijenhuis tensor $N_{J}$ is non-degenerate and that $J$ is not one of two $G_{2}$-invariant structures (thus $J$ is of types II or III). Then the (local and global) automorphisms of $J$ in the connected component of unity are only those coming from $G$, whence $\operatorname{dim} \operatorname{Aut}(M, J)=9$.

Some calculations from this work used symbolic packages of Maple; the corresponding worksheets are available from the authors.

\section{Classification Result Via RePresentation theOry}

Consider a homogeneous manifold $M=G / H$, i.e. a connected manifold $M$ on which a connected Lie group $G$ acts transitively with the stabilizer $H$ of a point $o \in M$. We will always assume that $G$ acts effectively on $M$, i.e. no non-trivial subgroup of $H$ is normal in $G$ (in non-effective case we can quotient by the kernel of the action).

In this case the isotropy representation $j: H \rightarrow \mathrm{GL}\left(T_{o} M\right)$ is almost faithful (has finite kernel) provided the stabilizer group $H$ is reductive (in particular, semi-simple that is our running assumption). When $M$ has a $G$-invariant almost complex structure $J$ whose Nijenhuis tensor $N_{J}$ is non-degenerate, then this is also the case by $\mathrm{K}_{2}$.

Let $\mathfrak{g}, \mathfrak{h}$ be the Lie algebras of the Lie groups $G, H$ and $\mathfrak{m}=T_{o} M$ the model tangent space of $G / H, o=e H$. The isotropy representation makes the space $\mathfrak{m}$ into $\mathfrak{h}$-module. The above data $(\mathfrak{h}$ subalgebra, $\mathfrak{m}$ representation) can be summarized in the following exact 3-sequence of $\mathfrak{h}$-modules

$$
0 \rightarrow \mathfrak{h} \longrightarrow \mathfrak{g} \longrightarrow \mathfrak{m} \rightarrow 0
$$

Using our hypothesis that $\mathfrak{h}$ is semi-simple yields the splitting of the sequence: we can find an embedding $\mathfrak{m} \subset \mathfrak{g}$ as an $H$-invariant complement. Thus we get the reductive decomposition

$$
\mathfrak{g}=\mathfrak{h}+\mathfrak{m}, \quad[\mathfrak{h}, \mathfrak{h}] \subset \mathfrak{h}, \quad[\mathfrak{h}, \mathfrak{m}] \subset \mathfrak{m} .
$$

Our strategy for classification is to start with the pure algebraic data, and then reconstruct the Lie groups $G, H$ and the manifold $M$ with its geometry (almost complex structure $J$ etc).

Reconstructing $\mathfrak{g}=\mathfrak{h} \oplus \mathfrak{m}$ from the representation $(\mathfrak{h}, \mathfrak{m})$ amounts to the following. The brackets $\mathcal{B}_{\mathfrak{h}}: \Lambda^{2} \mathfrak{h} \rightarrow \mathfrak{h}$ and $\mathcal{B}_{\mathfrak{h}, \mathfrak{m}}: \mathfrak{h} \wedge \mathfrak{m} \rightarrow \mathfrak{m}$ are given by the Lie algebra structure of $\mathfrak{h}$ and the $\mathfrak{h}$-module structure of $\mathfrak{m}$ respectively. The only missing ingredient is the bracket $\mathcal{B}_{\mathfrak{m}}: \Lambda^{2} \mathfrak{m} \rightarrow \mathfrak{g}$, and it determines the full bracket

$$
\mathcal{B}=\mathcal{B}_{\mathfrak{h}}+\mathcal{B}_{\mathfrak{h}, \mathfrak{m}}+\mathcal{B}_{\mathfrak{m}}: \Lambda^{2} \mathfrak{g}=\Lambda^{2} \mathfrak{h} \oplus(\mathfrak{h} \wedge \mathfrak{m}) \oplus \Lambda^{2} \mathfrak{m} \rightarrow \mathfrak{g}=\mathfrak{h} \oplus \mathfrak{m}
$$


Lemma 1. The Jacobi identity of the resulting bracket $\mathcal{B}: \Lambda^{2} \mathfrak{g} \rightarrow \mathfrak{g}$, involving an element from $\mathfrak{h}$, is equivalent to $\mathfrak{h}$-equivariancy of $\mathcal{B}_{\mathfrak{m}}$. If $\mathcal{B}_{\mathfrak{m}}$ is $\mathfrak{h}$-equivariant, then the bracket $\mathcal{B}$ defines the Lie algebra structure on $\mathfrak{g}$ iff the Jacobi map $\mathrm{Jac}_{\mathfrak{m}}: \Lambda^{3} \mathfrak{m} \rightarrow \mathfrak{g}$ vanishes, where

$$
\operatorname{Jac}_{\mathfrak{m}}(x, y, z)=\mathcal{B}\left(x, \mathcal{B}_{\mathfrak{m}}(y, z)\right)+\mathcal{B}\left(y, \mathcal{B}_{\mathfrak{m}}(z, x)\right)+\mathcal{B}\left(z, \mathcal{B}_{\mathfrak{m}}(x, y)\right) .
$$

The Lie algebra $\mathfrak{g}$ defined by such $\mathcal{B}_{\mathfrak{m}}$ is called the Lie algebra extension of the $\mathfrak{h}$-module $\mathfrak{m}$.

Proof. The Jacobi relation involving 3 elements from $\mathfrak{h}, \mathrm{Jac}_{\mathfrak{h}}: \Lambda^{3} \mathfrak{h} \rightarrow \mathfrak{h}$, vanishes as $\mathfrak{h}$ is a Lie algebra. The Jacobi relation involving 2 elements from $\mathfrak{h}$ and 1 from $\mathfrak{m}$ vanishes as $\mathfrak{m}$ is an $\mathfrak{h}$-representation. Finally the Jacobi relation involving 1 element from $\mathfrak{h}$ and 2 from $\mathfrak{m}$ is precisely the equivariancy of the map $\mathcal{B}_{\mathfrak{m}}$.

Since $\mathfrak{h}$ is semi-simple, the construction of $\mathcal{B}_{\mathfrak{m}} \in \operatorname{Hom}_{\mathfrak{h}}\left(\Lambda^{2} \mathfrak{m}, \mathfrak{g}\right)$ goes as follows. Decompose into irreducible $\mathfrak{h}$-modules (including the trivial): $\Lambda^{2} \mathfrak{m}=$ $\oplus r_{i} \cdot \mathfrak{u}_{i}=\oplus\left(\mathfrak{u}_{i} \otimes \mathbb{R}^{r_{i}}\right), \mathfrak{g}=\mathfrak{h} \oplus \mathfrak{m}=\oplus s_{i} \cdot \mathfrak{u}_{i}=\oplus\left(\mathfrak{u}_{i} \otimes \mathbb{R}^{s_{i}}\right)$. Then by Schur's lemma

$$
\operatorname{Hom}_{\mathfrak{h}}\left(\Lambda^{2} \mathfrak{m}, \mathfrak{g}\right)=\bigoplus \mathfrak{g l}_{\mathfrak{h}}\left(\mathfrak{u}_{\mathfrak{i}}\right) \otimes \operatorname{Hom}\left(\mathbb{R}^{r_{i}}, \mathbb{R}^{s_{i}}\right)=\oplus \operatorname{Mat}_{s_{i} \times r_{i}}
$$

where the space $\mathrm{Mat}_{s \times r}$ consists of real, complex or quaternionic $s \times r$ matrices (the algebra of splitting operators $\mathfrak{g l}_{\mathfrak{h}}\left(\mathfrak{u}_{\mathfrak{i}}\right)=\mathbb{R}, \mathbb{C}$ or $\mathbb{H}$ ).

Thus $\mathfrak{h}$-equivariancy of $\mathcal{B}_{\mathfrak{m}}$ can be effectively checked via the representation theory. On the contrary, vanishing of $\operatorname{Jac}_{\mathfrak{m}}\left(\Lambda^{3} \mathfrak{m}\right)$ is a set of linear and quadratic relations on $\mathcal{B}_{\mathfrak{m}}$ to be checked directly (representation theory can help here too: as $\mathrm{Jac}_{\mathfrak{m}}$ is $\mathfrak{h}$-equivariant, we can decompose $\Lambda^{3} \mathfrak{m}, \mathfrak{g}$ into irreducibles and apply Schur's lemma).

Invariant almost complex structures $J$ on $M$ bijectively corresponds to $\mathfrak{h}$-invariant tensors (here endomorphisms 2 )

$$
J \in\left(\mathfrak{m}^{*} \otimes \mathfrak{m}\right)^{\mathfrak{h}}=\operatorname{End}_{\mathfrak{h}}(\mathfrak{m}) \text { with } \quad J^{2}=-\mathbf{1} .
$$

Similarly, invariant pseudo-Riemannian metrics and almost symplectic structures on $M$ are in bijective correspondence with non-degenerate $\mathfrak{h}$-invariant tensors $g \in\left(S^{2} \mathfrak{m}^{*}\right)^{\mathfrak{h}}$ and $\omega \in\left(\Lambda^{2} \mathfrak{m}^{*}\right)^{\mathfrak{h}}$ respectively.

Our aim is to classify 6 -dimensional homogeneous manifolds $M=G / H$ with semisimple $H$, admitting an invariant almost complex structure $J$. Let $\mathfrak{g}=\mathfrak{h}+\mathfrak{m}$ be the associated reductive decomposition. By effectivity the isotropy representation ad $: \mathfrak{h} \rightarrow \mathfrak{g l}(\mathfrak{m})$ is exact (due to this all elements of $\mathfrak{g}$ act as non-trivial symmetries) and it preserves the complex structure $J$ on $\mathfrak{m}$. Therefore we identify $\mathfrak{h} \subset \mathfrak{g l}(\mathfrak{m}, J) \simeq \mathfrak{g l}_{3}(\mathbb{C})$.

\footnotetext{
${ }^{2}$ Endomorphisms are $\mathbb{R}$-linear and $\mathfrak{h}$-equivariant transformations of the module.
} 
2.1. Classification result. Our strategy is the following:

1. Enumerate all semi-simple subalgebras $\mathfrak{h} \subset \mathfrak{g l}_{3}(\mathbb{C})$, hence, all 6-dimensional $\mathfrak{h}$-modules $\mathfrak{m}$ with an invariant complex structure $J$.

2. Describe all $\mathfrak{h}$-equivariant linear maps $\mathcal{B}_{\mathfrak{m}}: \Lambda^{2} \mathfrak{m} \rightarrow \mathfrak{g}$ by decomposing the module $\Lambda^{2} \mathfrak{m}$ into irreducible submodules.

3. Compute all Lie algebra extensions $\mathfrak{g}$ of the $\mathfrak{h}$-module $\mathfrak{m}$ by solving the equations $\mathrm{Jac}_{\mathfrak{m}}=0 \in \Lambda^{3} \mathfrak{m}^{*} \otimes \mathfrak{g}$ on the parameters in $\mathcal{B}_{\mathfrak{m}}$.

4. Determine the homogeneous almost complex manifolds $M=G / H$ associated with the Lie algebra $\mathfrak{g}=\mathfrak{h}+\mathfrak{m}$ and the complex structure $J$.

The trivial bracket $\mathcal{B}_{\mathfrak{m}}=0$ defines the semidirect product Lie algebra $\mathfrak{g}=\mathfrak{h} \ltimes \mathbb{C}^{3}$ corresponding to the manifold $M=\mathbb{C}^{3}$ with the standard complex structure and the obvious action of the semi-direct Lie group $G=H \ltimes \mathbb{C}^{3}$. We call such structure flat and exclude them from consideration.

Below we use the following notations. For a classical simple Lie algebra $\mathfrak{h}$ denote by $V$ the standard (tautological) $\mathfrak{h}$-module. It has the natural complex structure if $\mathfrak{h}$ is $\mathfrak{s l}_{2}(\mathbb{C}), \mathfrak{s l}_{3}(\mathbb{C}), \mathfrak{s u}(3)$ or $\mathfrak{s u}(2,1)$. For $\mathfrak{h}=\mathfrak{s u}(2)$ we identify the module $V$ with the space $\mathbb{H}$ of quaternions and the algebra $\mathfrak{h}$ with imaginary quaternions $\operatorname{Im}(\mathbb{H})$ acting from the left. Similarly for $\mathfrak{h}=\mathfrak{s u}(1,1)$ we identify the module $V$ with the space $\mathbb{H}_{s}$ of split quaternions and the algebra $\mathfrak{h}$ with split imaginary quaternions $\operatorname{Im}\left(\mathbb{H}_{s}\right)$ acting from the left. The space of invariant complex structures on $V$ consists of right multiplications $R_{q}: x \mapsto x q$ by a quaternion $q$ with $q^{2}=-1$. So they are parametrized by the unit sphere $\mathbb{S}^{2} \subset \operatorname{Im}(\mathbb{H})$ in the first case and the unit pseudosphere $\mathbb{S}^{1,1} \subset \operatorname{Im}\left(\mathbb{H}_{s}\right)$ in the second case.

We identify the trivial 2-dimensional representation of $\mathfrak{h}$ and a complex structure $J$ with the standard pair $(\mathbb{C}, i)$. We denote by ad the adjoint representation of the Lie algebra $\mathfrak{h}$. If $\mathfrak{h}$ is real, the invariant complex structures on the module $\mathfrak{a d}^{\mathbb{C}}=\mathfrak{a} \mathfrak{d} \oplus \mathfrak{a} \mathfrak{d}$ are parametrized by

$$
J(\mathbf{v}, \mathbf{w})=\left(r \mathbf{v}-\frac{1+r^{2}}{t} \mathbf{w}, t \mathbf{v}-r \mathbf{w}\right),
$$

the same concerns the complexified tautological representation $V^{\mathbb{C}}$ of $\mathfrak{s l}_{3}(\mathbb{R})$.

Theorem 3. There are 7 different real semi-simple subalgebras in the complex Lie algebra $\mathfrak{g l}_{3}(\mathbb{C})$ (up to conjugation):

- $\mathfrak{h}=\mathfrak{s u}(2)$ or $\mathfrak{s u}(1,1)$, representations $V+\mathbb{C}, \mathfrak{a d}^{\mathbb{C}}$

- $\mathfrak{h}=\mathfrak{s l}_{2}(\mathbb{C})$, representations $V+\mathbb{C}, \mathfrak{a} \mathfrak{d}$;

- $\mathfrak{h}=\mathfrak{s l}_{3}(\mathbb{R})$, representation $V^{\mathbb{C}}$;

- $\mathfrak{h}=\mathfrak{s u}(3)$ or $\mathfrak{s u}(2,1)$ or $\mathfrak{s l}_{3}(\mathbb{C})$, representation $V$.

For the cases of $\mathfrak{s u}(2), \mathfrak{s u}(1,1)$ the possible Lie algebras $\mathfrak{g}$ with the specified representations $\mathfrak{m}$ are tabulated in the Appendix. For $\mathfrak{s l}_{2}(\mathbb{C})$ the adjoint representation gives only $\mathfrak{g}=\mathfrak{s l}_{2}(\mathbb{C}) \oplus \mathfrak{s l}_{2}(\mathbb{C})$ (so that $M=\mathrm{SL}_{2}(\mathbb{C}) \oplus$ $\mathrm{SL}_{2}(\mathbb{C}) / \mathrm{SL}_{2}^{\text {diag }}(\mathbb{C})$ ), while $V+\mathbb{C}$ leads to 2 cases for $\mathfrak{g}$ from the Appendix. For $\mathfrak{s u}(3)$ the corresponding $\mathfrak{g}$ is the Lie algebra of the exceptional group $G_{2}^{c}$. For $\mathfrak{s u}(2,1)$ the corresponding $\mathfrak{g}$ is the Lie algebra of the exceptional group 
$G_{2}^{*}$. The other cases $\mathfrak{s l}_{3}(\mathbb{R})$ and $\mathfrak{s l}_{3}(\mathbb{C})$ give only the flat structures (in which case $M^{6}=\mathbb{C}^{3}$ or its quotient).

The proof of this theorem is a straightforward (but lengthy) calculation, we sketch it in the next section.

2.2. Proof of Theorem 1. On the level of Lie algebras Theorem 1 follows instantly from Theorem 3 and the tables from the Appendix.

The passage to the Lie groups is straightforward for types I and II, because in these cases we indicate the pair $(G, H)$, and it remains to treat only different discrete quotients. But for type III we need to establish existence of the Lie group $G$ such that $\mathfrak{h}$ exponentiates into its Lie subgroup. In general for a Lie groups $H$ with a homomorphic embedding of Lie algebras $\iota: \mathfrak{h}=\operatorname{Lie}(H) \subset \mathfrak{g}$ there does not exist a Lie group $G$ with $\mathfrak{g}=\operatorname{Lie}(G)$ and a homomorphic embedding $H \hookrightarrow G$ having differential $\iota$, see counter-examples and discussion in [B, GOV].

In our case, however the Lie functor works nicely. The output of Theorem 3 yields the structure of Lie algebra on $\mathfrak{g}=\mathfrak{h}+\mathfrak{m}$, and we should consider the cases, when $\mathfrak{m}$ is closed with respect to these brackets, i.e. [, $]_{\mathfrak{m}}^{\mathfrak{h}}=0$ in terms of splitting of the bracket $\mathcal{B}_{\mathfrak{m}}$. Indeed, in the cases when $\mathfrak{m} \subset \mathfrak{g}$ is not a Lie subalgebra (types I and II in Theorem 1) we have an obvious Lie subgroup $H \subset G$ such that $M=G / H$ is the required homogeneous almost complex manifold.

Proposition 1. The pairs $(\mathfrak{g}, \mathfrak{h})$ of Lie algebra/subalgebra from the Tables of the Appendix with $\mathfrak{m}$ being a Lie algebra correspond to the pairs $(G, H)$ of Lie group/subgroup of type III in Theorem 1 .

This statement concerns the cases A1-A6 from the Tables except for the cases A1.4 and A3.5, which correspond to type II (case A6 is rather simple and was already discussed in Theorem 3 ).

Proof. Let $M$ be the simply connected Lie group corresponding to $\mathfrak{m}$ (for the representation $V+\mathbb{C}$ the Lie algebra $\mathfrak{m}$ is solvable and so $M \simeq \mathbb{R}^{6}$ as a manifold, for the representation $\mathfrak{a d}^{\mathbb{C}}$ the choice of $M$ is obvious). Consider the representation $\rho: \mathfrak{h} \rightarrow \operatorname{End}(\mathfrak{m})$. Then there exists a homomorphism $R: H \rightarrow G L(\mathfrak{m})$ such that $d R=\rho$. By virtue of Proposition 4.2 of $[\mathrm{VO}$, Chapter 2] the semi-direct product $G=H \ltimes_{R} M$ is the desired simplyconnected Lie group. The main idea of this approach follows Cartan's proof of the third Lie theorem [C].

Another proof is based on the Palais' criterion $[\mathrm{P}]$ for a transformation group to be a Lie group. Namely, $M$ acts on itself by left translations and $H \subset \operatorname{Diff}(M)$ is a closed subgroup (as it is the stabilizer of the unity and closed in $G L\left(T_{e} M\right)$ ). Both $M$ and $H$ generate a closed subgroup in the group of diffeomorphisms, which is a Lie group $G$ with $\operatorname{Lie}(G)=\mathfrak{g}=\mathfrak{h} \ltimes \mathfrak{m}$.

Thus, when $\mathfrak{m} \subset \mathfrak{g}$ is a Lie subalgebra we also get representation $M=G / H$ and this finishes the proof of Theorem 1 . 


\section{Proof of the Classification RESUlt}

Representation part of Theorem 3 (list of 7 cases) is obvious from the general theory of representations of semi-simple Lie algebras. The hard part is the reconstruction of possible Lie brackets on $\mathfrak{g}=\mathfrak{h}+\mathfrak{m}$.

Let us consider the first of the cases, when $\mathfrak{h}=\mathfrak{s u}(2)$ and the isotropy as a complex representation has type $\mathfrak{m}=V+\mathbb{C}$. We can identify $\mathfrak{h}$ with the Lie algebra of imaginary quaternions $\operatorname{Im}(\mathbb{H})$ and $V$ with the left $\mathfrak{h}$-module $\mathbb{H}$. The endomorphism ring of $V$ is the algebra $\mathbb{H}$ acting from the right (we will use this freedom to change the basis in $V$ ). The following easy claim will be used repeatedly.

Lemma 2. The $\mathfrak{h}$-invariant complex structure $J$ on the module $\mathfrak{m}=V+\mathbb{C}$ is given by the formula

$$
J(x, \eta)=(x q, i \eta), \quad x \in V=\mathbb{H}, \eta \in \mathbb{C},
$$

where $q \in \mathbb{S}^{2} \subset \operatorname{Im}(\mathbb{H})$ is a unit imaginary quaternion.

It is possible to fix $q$ to be equal to $i \in \mathbb{H}$ by an endomorphism, but as noticed above we use this freedom to simplify the brackets. On the other hand, though on the 2-dimensional trivial $\mathfrak{h}$-module $\mathbb{R}^{2}$ there is a 2-parametric family of complex structures, we fix one (equal to $i$, turning this submodule into $\mathbb{C}$ ) as this freedom does not help to simplify the brackets.

The first task in constructing the map $\mathcal{B}_{\mathfrak{m}}$ is to decompose the $\mathfrak{h}$-module $\Lambda^{2} \mathfrak{m}=\Lambda^{2}(V+\mathbb{C})=\Lambda^{2} V+V \otimes \mathbb{C}+\Lambda^{2} \mathbb{C}$ into irreducibles. From the representation viewpoint (without complex structure $J$ ) $\mathbb{C}$ is the trivial 2dimensional real module $\mathbb{R}^{2}$, so $\Lambda^{2} \mathbb{C} \simeq \mathbb{R}^{1}$ and $V \otimes \mathbb{C}=V+V$.

Lemma 3. As an $\mathfrak{h}$-module $\Lambda^{2} V=\mathfrak{a} \mathfrak{d}+\mathbb{R}^{3}$.

Proof. Let us give two proofs, one via the representation theory of simple Lie algebras and the other straightforward.

The complexified Lie algebra $\mathfrak{h}^{\mathbb{C}}=\mathfrak{s l}(2, \mathbb{C})$ has the same standard representation $V$ (it is not absolutely irreducible). Changing the real form to $\mathfrak{h}^{\prime}=\mathfrak{s u}(1,1) \simeq \mathfrak{s l}(2, \mathbb{R})$ we obtain by the highest weight decomposition $V=W+W$, where $W$ is the standard representation of $\mathfrak{s l}(2, \mathbb{R})$. Now $\Lambda^{2}(W+W)=\Lambda^{2}(W) \otimes \mathbb{R}^{2}+W \otimes W=S^{2} W+\mathbb{R}^{3}=\mathfrak{a} \mathfrak{d}+\mathbb{R}^{3}$. This induces the above decomposition.

A more direct proof is as follows. Let us identify $V \simeq V^{*}$ using the $\mathfrak{h}$ invariant metric on $V=\mathbb{H}: g(x, y)=\operatorname{Re}(x \bar{y}), x, y \in V$. Consider the 2-forms $\omega_{b}, \omega^{b} \in \Lambda^{2} V^{*}$ given by

$$
\omega_{b}(x, y)=\operatorname{Re}(x b \bar{y}), \quad \omega^{b}(x, y)=\operatorname{Re}(x \bar{y} b), \quad b \in \operatorname{Im}(\mathbb{H})
$$

(check both are skew-symmetric!).

The group $H=S U(2) \simeq \mathbb{S}^{2} \subset \operatorname{Im}(\mathbb{H})$ acts on them as follows $(q \in H)$

$$
\begin{gathered}
\omega_{b}(q x, q y)=\operatorname{Re}(q x b \overline{q y})=\operatorname{Re}(q x b \bar{y} \bar{q})=\operatorname{Re}\left(x b \bar{y}|q|^{2}\right)=\omega_{b}(x, y), \\
\omega^{b}(q x, q y)=\operatorname{Re}(q x \overline{q y} b)=\operatorname{Re}(q x \bar{y} \bar{q} b)=\operatorname{Re}\left(x \bar{y} q^{-1} b q\right)=\omega_{\operatorname{Ad}_{q}^{-1} b}(x, y) .
\end{gathered}
$$


Consequently 6-dimensional $\Lambda^{2} V^{*}$ has two 3-dimensional submodules $\left\{\omega^{b}\right\}$ and $\left\{\omega_{b}\right\}$. By the above the first of them has type $\mathfrak{a} \mathfrak{d}$ and the second is trivial $\mathbb{R}^{3}$. Thus they do not intersect and so span the whole $\Lambda^{2} V^{*}$. The lemma is proved.

Lemma 4. Let $\mathfrak{g}=\mathfrak{a} \mathfrak{d}+V+\mathbb{C}$ be a Lie algebra extension of the $\mathfrak{h}$-module $V+\mathbb{C}$. Then the brackets on $\mathfrak{m}$ are

$$
[V, V] \subset \mathfrak{a} \mathfrak{d}+\mathbb{C}, \quad[V, \mathbb{C}] \subset V, \quad[\mathbb{C}, \mathbb{C}] \subset \mathbb{C} .
$$

Here from the naked representation theory viewpoint $\mathbb{C}=\mathbb{R}^{2}$, but we shall use the structure $i$ on it. The lemma has the following implications.

- $\mathbb{C}$ is a Lie subalgebra of $\mathfrak{g}$ and it is solvable: $\Lambda^{2} \mathbb{C} \mapsto \mathbb{R} \subset \mathbb{C}$. There exists a $J$-adapted basis e, $i$ e of $\mathbb{C}$ such that $[\mathrm{e}, i \mathrm{e}]=\varepsilon \mathrm{e}$ for some $\varepsilon \in\{0,1\}$ (for $\varepsilon=1$ this determines e uniquely).

- Since $\mathbb{C}$ is the trivial $\mathfrak{h}$-module, the bracket $V \otimes \mathbb{C} \rightarrow V$ is composed of endomorphisms, i.e. $[x, \eta]=A_{\eta}(x)$, where $\eta \mapsto-A_{\eta}$ is the homomorphism of Lie algebras $\mathbb{C} \rightarrow \operatorname{Lie}\left(\operatorname{End}_{\mathbb{R}}(V)\right)=\mathbb{R} \oplus \mathfrak{h} \simeq \mathfrak{u}(2)$. Since the latter has no non-trivial solvable subalgebras, the homomorphism is not injective when the subalgebra $\mathbb{C}$ is not abelian $\left(\varepsilon=1 \Rightarrow A_{\mathrm{e}}=0\right)$.

- The $V$-bracket $\Lambda^{2} V \rightarrow \mathfrak{g}$ for some $\lambda \in \mathbb{R}, b, b^{\prime} \in \operatorname{Im}(\mathbb{H})$ equals

$$
[x, y]=\lambda \cdot \operatorname{Im}(x \bar{y})+\left(\omega_{b}(x, y) \mathrm{e}+\omega_{c}(x, y) i \mathrm{e}\right)
$$

Elaborating upon Lemma 1 with these choices $\mathfrak{g}$ is a Lie algebra iff the $V$ Jacobi identity holds $-\operatorname{Jac}_{\mathfrak{m}}: \Lambda^{3} V \rightarrow \mathfrak{g}$ is zero and in addition

$$
\begin{aligned}
& \omega_{b}\left(A_{i \mathrm{e}} x, y\right)+\omega_{b}\left(x, A_{i \mathrm{e}} y\right)=\varepsilon \omega_{b}(x, y), \\
& \omega_{b}\left(A_{\mathrm{e}} x, y\right)+\omega_{b}\left(x, A_{\mathrm{e}} y\right)=-\varepsilon \omega_{c}(x, y), \\
& \omega_{c}\left(A_{\eta} x, y\right)+\omega_{c}\left(x, A_{\eta} y\right)=0, \quad \eta \in \mathbb{C} .
\end{aligned}
$$

It is also important to notice that since $\mathbb{H}$ is a division algebra, then every nonzero operator $A_{\eta} \in \operatorname{End}_{\mathbb{R}}(V)=\mathbb{H}$ is invertible. Now we consider the following possibilities (if $\lambda=0$, then $\mathfrak{m}$ is a Lie algebra).

(A1.1) $\lambda=0,[V, V] \neq 0$ and the subalgebra $A_{\mathbb{C}} \subset \mathbb{H}$ is nonzero. We claim that the map $A$ has a kernel. Indeed, for $\varepsilon=1$ we have $A_{\mathrm{e}}=0$. For $\varepsilon=0$ denoting $A_{\eta}(x)=x q, q \in \mathbb{H}$, then (2) implies $\operatorname{Re}(x(q d+d \bar{q}) \bar{y})=0$, where $d$ is any linear combination of $b, c \in \operatorname{Im}(\mathbb{H})$. As $b, c$ are not simultaneously zero, this yields a kernel, which can be accommodated into e (using $G L(1, \mathbb{C}$ )freedom of change of basis in the $\mathbb{C}$-module for $\varepsilon=0$ ).

Then the Jacobi identity $\operatorname{Jac}_{\mathfrak{m}}\left(\Lambda^{3} V\right)=0$ and the remark about invertibility imply that $c=0$. Using the endomorphism freedom in the choice of basis in $V$, we can assume $b=\alpha i$. Using again (2) we obtain $A_{i \mathrm{e}}(x)=x\left(\frac{\varepsilon}{2}+r i\right)$ for some $r \in \mathbb{R}$. Thus in this case $\mathfrak{m}$ is a Lie algebra with the structure 
equations 3

$$
[x, y]=\alpha \operatorname{Re}(x i \bar{y}) \mathrm{e},[x, i \mathrm{e}]=x\left(\frac{\varepsilon}{2}+r i\right),[\mathrm{e}, i \mathrm{e}]=\varepsilon \mathrm{e} .
$$

In other words, $\mathfrak{m}$ is obtained in two steps. First we construct the central extension of abelian algebra $V$ by the 2-form $\omega_{i}$ - we get the Heisenberg Lie algebra $\mathfrak{h e i s}(V)=V+\mathbb{R e}$. Then we take its 1-dimensional extension of by the derivation $\mathrm{ad}_{i \mathrm{e}}$. In Table A1 this case is called A1.1.

(A1.2) $\lambda=0,[V, V] \neq 0$ and the subalgebra $A_{\mathbb{C}} \subset \mathbb{H}$ is zero. In this case the subalgebra $\mathbb{C}$ must be abelian, and then the Jacobi identity holds $\operatorname{Jac}_{\mathfrak{m}}\left(\Lambda^{3} V\right)=0$ identically. We can normalize (by rescaling e) $b=i$ and $c=p \in \operatorname{Im}(\mathbb{H})$ is arbitrary.

Thus $\mathfrak{m}$ is the Lie algebra, which is a 2-dimensional central extension of abelian $V$ by two 2 -forms $\omega_{i}, \omega_{p}$. This is the case A1.2 of Table A1.

(A1.3) $\lambda=0,[V, V]=0$. Here the Jacobi identity is satisfied and we only need to normalize the map $\eta \mapsto A_{\eta}$. If $\varepsilon \neq 0$, then $A_{\mathrm{e}}=0$ and we choose a basis in $V$ such that $A_{i \mathrm{e}}(x)=x(\beta+r i), \beta, r \in \mathbb{R}$.

On the other hand, if $\varepsilon=0$, then $A_{\mathbb{C}} \subset \mathbb{H}$ is either 1-dimensional or 2-dimensional subalgebra, which is possible only for $A_{\mathrm{e}}(x)=x \alpha, A_{i \mathrm{e}}(x)=$ $x(\beta+r i), \alpha, \beta, r \in \mathbb{R}$. Clearly we can normalize $\alpha=0$ or 1 .

Thus $\mathfrak{m}$ is a Lie algebra, which is either an extension of abelian $V$ by two commuting derivations $\operatorname{ad}_{\mathrm{e}}, \operatorname{ad}_{i \mathrm{e}}$ or an extension of abelian $V+\mathbb{R e}$ by one derivation $\operatorname{ad}_{i \mathrm{e}}$. This is the case A1.3 of Table A1.

(A1.4) $\lambda \neq 0$. Here $\mathfrak{m}$ is not a Lie subalgebra of $\mathfrak{g}$. In this case the Jacobi identity implies that $\mathbb{C}$ has a central element (in particular $\mathbb{C}$ is abelian). We can choose it to be $i$ e. Then $A_{i \mathrm{e}}=0, c=0$. We can normalize (by endomorphisms) $\lambda= \pm 1, b=i$. The Jacobi identity further yields $A_{\mathrm{e}}(x)=$ $3 \lambda x i$.

The obtained Lie algebra $\mathfrak{g}$ is isomorphic to $\mathfrak{u}_{3}$ for $\lambda=1$ and to $\mathfrak{u}_{1,2}$ for $\lambda=-1$. The associated almost complex manifolds are $U(3) / S U(2)$ and $U(2,1) / S U(2)$ respectively. In Table A1 this case is called A1.4.

Thus we obtained the complete classification of the homogeneous structures $M=G / H$ in the first case from the list of Theorem 3. Almost complex structures on $M$ are obtained from $\mathfrak{h}$-invariant complex structures on $\mathfrak{m}$ specified above.

The Nijenhuis tensor can be computed by the formula

$$
N_{J}(X, Y)=\pi([J X, J Y]-J[X, J Y]-J[J X, Y]-[X, Y]), \quad X, Y \in \mathfrak{m},
$$

\footnotetext{
${ }^{3}$ Here and in what follows we adopt the convention that $x, y \in V$ are arbitrary elements, but e $\in \mathbb{C}$ is a fixed element, in particular e, $i$ e is a real basis of $\mathbb{C}$.

4The central ("left") extension and extension by derivations ("right") $\tilde{\mathfrak{g}}$ of the Lie algebra $\mathfrak{g}$ (via $\mathfrak{f}$ ) are given respectively by the exact sequences

$$
0 \rightarrow \mathfrak{f} \rightarrow \tilde{\mathfrak{g}} \rightarrow \mathfrak{g} \rightarrow 0, \quad 0 \rightarrow \mathfrak{g} \rightarrow \tilde{\mathfrak{g}} \rightarrow \mathfrak{f} \rightarrow 0 .
$$

Then $\mathfrak{g}$ is respectively the quotient/subalgebra of $\tilde{\mathfrak{g}}$ and its bracket can/cannot change upon the extension.
} 
where [,] $: \Lambda^{2} \mathfrak{m} \rightarrow \mathfrak{g}$ are the brackets in the Lie algebra $\mathfrak{g}$ and $\pi: \mathfrak{g} \rightarrow \mathfrak{m}$ is the projection along $\mathfrak{h}$. Similarly the differential of the almost symplectic structure $\omega$ on $\mathfrak{m}$ is computed by the Cartan formula using only the brackets on $\mathfrak{m}$. This explains all entries in Table A1.

Consider the other representation $\mathfrak{m}=\mathfrak{a d}^{\mathbb{C}}$ of $\mathfrak{h}=\mathfrak{s u}(2)$. Here it is more convenient to involve Levi decomposition: $\mathfrak{g}=\mathfrak{g}_{s s} \oplus \mathfrak{r}$, where the first summand is a semi-simple part and the second is the radical. The factor $\mathfrak{g}_{s s}$ can be chosen to contain $\mathfrak{h}$, and so can be one of the following:

- $\mathfrak{g}_{s s}=\mathfrak{s u}(2)^{3}, \mathfrak{h}=\mathfrak{s u}(2)_{\operatorname{diag}}$,

- $\mathfrak{g}_{s s}=\mathfrak{s u}(2) \oplus \mathfrak{s l}(2, \mathbb{C}), \mathfrak{h}=\mathfrak{s u}(2)_{\text {diag }}$,

- $\mathfrak{g}_{s s}=\mathfrak{s u}(2)^{2}, \mathfrak{h}=\mathfrak{s u}(2)_{\text {diag }}$,

- $\mathfrak{g}_{s s}=\mathfrak{s l}(2, \mathbb{C}), \mathfrak{h} \subset \mathfrak{s l}(2, \mathbb{C})$.

The last case is disqualified as $\mathfrak{h}$ acts by zero on $\mathfrak{g} / \mathfrak{g}_{s s}$ (no 3-dimensional nontrivial representation for $\mathfrak{g}_{s s}$ ). The other give the cases A2.1, A2.2, A2.3 of Table A2 respectively.

Finally, it is possible that $\mathfrak{g}_{s s}=\mathfrak{h}$, whence $\mathfrak{m}=\mathfrak{r}$. Since this is solvable of module type $\mathfrak{a d}^{\mathbb{C}}$, the only non-trivial brackets in terms of the splitting $\mathfrak{m}=\mathfrak{m}_{1}+\mathfrak{m}_{2}$ (grading) are the following (case A2.4)

$$
\left[x_{1}, y_{1}\right]=[x, y]_{2}, \quad x, y \in \mathfrak{a} \mathfrak{d} \simeq \mathfrak{h} .
$$

The corresponding analysis for $\mathfrak{h}=\mathfrak{s u}(1,1)$ is similar (for instance, Lemma 2 holds true with $\mathbb{H}$ changed to $\mathbb{H}_{s}$ ), but a special care should be taken as in this non-compact case there are null elements on the representation $V$ with respect to its unique (up to scale) $\mathfrak{h}$-invariant metric (that's why Table A3 is bigger than A1). The cases $\mathfrak{h}=\mathfrak{s l}_{2}(\mathbb{C}), \mathfrak{s l}_{3}(\mathbb{R}), \mathfrak{s l}_{3}(\mathbb{C})$ are much simpler. The details of computations can be found in [Wi]. For the largest algebras $\mathfrak{s u}(3)$ and $\mathfrak{s u}(2,1)$ arising in the symmetry analysis the computations are done in $\left[\mathrm{K}_{2}\right]$.

This finishes the proof of Theorem 3 .

\section{Automorphism groups of nondegenerate structures $J$}

Here we prove Theorem 2 - find the symmetry algebra of the structures $J$ with non-degenerate Nijenhuis tensor $N_{J}$. It follows from the tables that $N_{J}$ can be non-degenerate only for isotropy algebras $\mathfrak{s u}(2), \mathfrak{s u}(1,1)$ or $\mathfrak{s u}(3)$, $\mathfrak{s u}(2,1)$. In the latter two cases $J$ is locally isomorphic to the $G_{2}$-invariant almost complex structure on either $S^{6}$ or $S^{2,4}$ and if the automorphism group has dimension 14 , it is one of the two forms of the group $G_{2}$, see $\mathrm{K}_{2}$ for details. In what follows we consider the former two cases.

According to $\mathrm{K}_{2}$ the isotropy algebra $\mathfrak{s y m}(J)_{o}$ of the symmetry algebra $\mathfrak{s y \mathfrak { m }}(J)$ at the point $o \in M$ is 1 -jet determined. Indeed, the proof of Theorem 1 loc.cit. implies

Theorem 4. If the Nijenhuis tensor $N_{J}$ on a connected almost complex manifold $\left(M^{6}, J\right)$ is non-degenerate, then any vector field $X \in \mathfrak{s y m}(J)$, is uniquely 
determined by its 1-jet $[X]_{o}^{1}$. Consequently, the isotropy algebra satisfies:

$$
\mathfrak{s y \mathfrak { m }}(J)_{o}=\left\{X: L_{X}(J)=0, X(o)=0\right\} \subset \mathfrak{g l}(\mathfrak{m}, J) .
$$

4.1. Symmetries via derivations. Theorem 4 hints to the following statement concerning the symmetry algebra of the homogeneous models A1.1, A2, A3.1, A3.2 and A4 according to the numeration in Appendix.

Proposition 2. Let $M=G / H$ be the homogeneous almost complex manifold associated with one of the Lie algebra extensions of the $\mathfrak{h}$-module $\mathfrak{m}$ in the case when $\mathfrak{m}$ is a Lie algebra (subalgebra in $\mathfrak{g}$ ) and $\mathfrak{h}$ is either $\mathfrak{s u}(2)$ or $\mathfrak{s u}(1,1)$. If the Nijenhuis tensor $N_{J}$ of the almost complex structure $J$ is non-degenerate, then the full symmetry algebra as a vector space is

$$
\mathfrak{s y \mathfrak { m }}(J)=\mathfrak{m}+\mathfrak{s y \mathfrak { m }}(J)_{o}
$$

and the full isotropy algebra equals

$$
\mathfrak{s y \mathfrak { m }}(J)_{o}=\mathfrak{d e r}(\mathfrak{m}) \cap \mathfrak{g l}(\mathfrak{m}, J)=\{A \in \mathfrak{d e r}(\mathfrak{m}): A J=J A\} .
$$

Otherwise said, we are given the pair $(\mathfrak{g}, \mathfrak{h})$ with $\mathfrak{m}=\mathfrak{g} / \mathfrak{h}$ and $\mathfrak{h}$-invariant $J$ on $\mathfrak{m}$. The claim is that if we can find an extension $(\tilde{\mathfrak{g}}, \tilde{\mathfrak{h}}) \supset(\mathfrak{g}, \mathfrak{h})$ with the same property, then still $\tilde{\mathfrak{h}}$ acts on $\mathfrak{m}$ by derivations.

Below we denote by $\pi: \tilde{\mathfrak{g}} \rightarrow \mathfrak{m}$ the projection along $\tilde{\mathfrak{h}}$ and use the labels for almost complex homogeneous spaces from the Appendix.

Proof. Let $\tilde{\mathfrak{g}}=\mathfrak{s y \mathfrak { m }}(J)$ be the full symmetry algebra. By $\left[\mathrm{K}_{1}, \mathrm{~K}_{2}\right.$ the full isotropy algebra $\tilde{\mathfrak{h}}=\mathfrak{s y m}(J)_{o} \subset \tilde{\mathfrak{g}}$ is at most 8-dimensional. If $J$ is not locally isomorphic to the $G_{2}^{c}$-invariant almost complex structure on $\mathbb{S}^{6}$ or $G_{2}^{*}$-invariant almost complex structure on $S^{2,4}$, then $\operatorname{dim} \tilde{\mathfrak{h}} \leq 5$. Indeed, this is so in any non-exceptional case of canonical forms NDG(1-4) of $\left[K_{1}\right.$, exceptions are the two cases given by formulae (16) and (17) of $\left[\mathrm{K}_{2}\right.$, Section 7] when $\tilde{\mathfrak{h}}$ is equal to $\mathfrak{s u}(2,1)$ or $\mathfrak{s u}(3)$ respectively. The structure of $\mathfrak{g}$ recovers uniquely and $M=G_{2}^{*} / S U(2,1)$ in the first case and $M=G_{2}^{c} / S U(3)$ in the second case respectively; the structure $J$ in every case is unique. Any proper subalgebra of $\mathfrak{s u}(2,1)$ is at most 5 -dimensional, and that of $\mathfrak{s u}(3)$ is at most 4-dimensional, whence the claim.

Since already $J$ has 3 -dimensional isotropy $\mathfrak{h}$ by construction, the additional subspace $\mathfrak{r} \subset \tilde{\mathfrak{h}}$ has $\operatorname{dim} \mathfrak{r} \leq 2$. Thus this $\mathfrak{r}$ can be chosen the radical of the full isotropy algebra $\tilde{\mathfrak{h}} \subset \tilde{\mathfrak{g}}$.

We start with the $\mathfrak{a d}^{\mathbb{C}}$ representation of $\mathfrak{h}=\mathfrak{s u}(2)$ on $\mathfrak{m}$. Then by $\mathfrak{h}$ equivariance of the brackets, $\mathfrak{r}$ is in the radical $\tilde{\mathfrak{r}}$ of $\tilde{\mathfrak{g}}$ (this follows by dimensional reasons and Levi decomposition of $\mathfrak{r} \oplus \mathfrak{m}^{\prime}$, where $\mathfrak{m}^{\prime}$ is the part of $\mathfrak{m}$ that does not enter into the semi-simple part $\left.\mathfrak{g}_{s s} \subset \mathfrak{g}\right)$.

If $\mathfrak{m}$ is either $\mathfrak{s u}(2) \oplus \mathfrak{s u}(2)$ or $\mathfrak{s l}(2, \mathbb{C})(\mathrm{A} 2.1, \mathrm{~A} 2.2)$, then $\mathfrak{g}$ is semi-simple, so extension of $\mathfrak{h}$ to $\tilde{\mathfrak{h}}$ is by radical $\mathfrak{r}$ only, whence $[\mathfrak{r}, \mathfrak{m}] \subset \mathfrak{r}$ and hence the added summand $\mathfrak{r}$ acts non-effectively, which is prohibited.

In the case $\mathfrak{m}=\mathfrak{s u}(2)+\mathbb{R}^{3}$ (A2.3) the radical of $\tilde{\mathfrak{g}}$ is $\tilde{\mathfrak{r}}=\mathfrak{r}+\mathbb{R}^{3}$ and so we have: $[\mathfrak{r}, \mathfrak{s u}(2)]=0$ and $\left[\mathfrak{r}, \mathbb{R}^{3}\right] \subset \mathfrak{r}+\mathbb{R}^{3}$. By $J$-invariancy of $\pi \circ \mathfrak{a} \mathfrak{d}_{\mathfrak{r}}(\mathfrak{m})$ we 
get $\pi([\mathfrak{r}, \mathfrak{m}])=0$. This means that the action of $\mathfrak{r}$ on $\mathfrak{m}$ is non-effective and we exclude such $\mathfrak{r}$ as before.

In the last $\mathfrak{a d}^{\mathbb{C}}$-case $\mathfrak{m}=\mathfrak{a}_{1} \oplus \mathfrak{a}_{2}(\mathrm{~A} 2.4)$ the Lie algebra structure is graded and the $\mathfrak{h}$-representation on $\mathfrak{a}_{i} \simeq \mathbb{R}^{3}$ is adjoint. In the radical $\tilde{\mathfrak{r}}=\mathfrak{m}+\mathfrak{r}$ the $\mathfrak{h}$-representation $\mathfrak{r}$ is the trivial submodule, whence by $\mathfrak{h}$-equivariance $[\mathfrak{r}, \mathfrak{m}] \subset \mathfrak{m}$. This means that $\mathfrak{r}$ acts by derivations, as required in Proposition 2 .

The case $\mathfrak{h}=\mathfrak{s u}(1,1)$ is similar except for the last type $\mathfrak{m}=\mathfrak{a}_{1} \oplus \mathfrak{a}_{2}$. Then another possibility occurs that $\mathfrak{r}$ is the standard representation $\mathcal{R}\left(\lambda_{1}\right)$ of $\mathfrak{h} \simeq \mathfrak{s l}_{2}(\mathbb{R})$, where $\mathcal{R}(w)$ is the representation of highest weight $w$. As $\mathfrak{a}_{i}=\mathcal{R}\left(2 \lambda_{1}\right)$ and $\mathcal{R}\left(\lambda_{1}\right) \otimes \mathcal{R}\left(2 \lambda_{1}\right)=\mathcal{R}\left(\lambda_{1}\right)+\mathcal{R}\left(3 \lambda_{1}\right), \mathfrak{h}$-equivariance implies $[\mathfrak{r}, \mathfrak{m}] \subset \mathfrak{r}$, so the action is non-effective.

Consider now the second possible representation of $\mathfrak{h}: \mathfrak{m}=V+\mathbb{C}$. Starting with $\mathfrak{h}=\mathfrak{s u}(2)$ we note the additional subspace $\mathfrak{r}$ is the trivial $\mathfrak{h}$-module. Again $\mathfrak{h}$-equivariance (together with Schur's lemma) implies $[\mathfrak{r}, V] \subset V$. Since $0 \neq[V, V] \subset \mathbb{C}$ and $\left.\pi \circ \mathfrak{a d}_{\mathfrak{r}}\right|_{\mathfrak{m}}$ commutes with $J$, the Jacobi identity implies $[\mathfrak{r}, \mathbb{C}] \subset\langle[V, V], J[V, V]\rangle=\mathbb{C}$. Thus $[\mathfrak{r}, \mathfrak{m}] \subset \mathfrak{m}$ and $\mathfrak{r}$ acts by derivations, as claimed in Proposition 2 ,

When $\mathfrak{h}=\mathfrak{s u}(1,1)$ and $\mathfrak{r}$ as $\mathfrak{h}$-representation is trivial, the argument is the same (here $\operatorname{dim} \mathfrak{r} \leq 2$ ). Consider the case $\mathfrak{r} \simeq \mathbb{R}^{2}$ being the standard representation $U$ of $\mathfrak{h} \simeq \mathfrak{s l}_{2}(\mathbb{R})$. In this case $V=U^{\mathbb{C}}$ is $2 \mathcal{R}\left(\lambda_{1}\right)$ as $\mathfrak{h}$ representation. Therefore as $\mathcal{R}\left(\lambda_{1}\right) \otimes \mathcal{R}\left(\lambda_{1}\right)=\mathcal{R}(0)+\mathcal{R}\left(2 \lambda_{1}\right)$ (where the first summand is the trivial 1-dimensional representation and the second is the adjoint), we get by $\mathfrak{h}$-equivariance $[\mathfrak{r}, V] \subset \mathfrak{h}+\mathbb{C}$. Since $0 \neq[V, V] \subset \mathbb{C}$, $[V, \mathbb{C}] \subset V,[\mathfrak{h}, V]=V$ and $\left.\pi \circ \mathfrak{a} \mathfrak{d}_{\mathfrak{r}}\right|_{\mathfrak{m}}$ commutes with $J$, the Jacobi identity yields $[\mathfrak{r}, \mathbb{C}] \subset[\mathfrak{h}+\mathbb{C}, V]=V$.

Let us show that $[\mathfrak{r}, V]$ does not have $\mathfrak{h}$ component. Indeed, since $[\mathfrak{r}, \mathfrak{r}] \subset$ $\mathfrak{h}+\mathbb{C}$ by $\mathfrak{h}$-equivariance, the Jacobi identity implies

$$
V \supset[[\mathfrak{r}, \mathfrak{r}], V] \subset[\mathfrak{r},[\mathfrak{r}, V]] \subset[\mathfrak{r}, \mathfrak{h}+\mathbb{C}] \subset \mathfrak{r}+V .
$$

Since $[\mathfrak{r}, \mathfrak{h}]=\mathfrak{r}$, the presence of $\mathfrak{h}$ component implies non-trivial $\mathfrak{r}$ component in the last summand of the above display formula. To study it consider the bracket-maps $\psi: \mathfrak{r} \times \mathfrak{h} \rightarrow \mathfrak{r}$ and $\phi: \mathfrak{r} \times V \rightarrow \mathfrak{h}$ (for the latter we postcompose with the projection). The Jacobi identity and the above display formula imply $\psi\left(r_{1}, \phi\left(r_{2}, v\right)\right)=\psi\left(r_{2}, \phi\left(r_{1}, v\right)\right)$ for all $r_{1}, r_{2} \in \mathfrak{r}, v \in V$. Since the maps $\psi, \phi$ depend only on the $\mathfrak{h}$-module structure and as such $\mathfrak{r}=U$, $V=U \oplus U$, we change the maps to

$$
\Psi: U \times \mathfrak{h} \rightarrow U, \Phi: U \times U \rightarrow \mathfrak{h} \text { with } \Psi\left(u_{1}, \Phi\left(u_{2}, u_{3}\right)\right)=\Psi\left(u_{2}, \Phi\left(u_{1}, u_{3}\right)\right) .
$$

Here $\Psi$ is the standard representation, and $\Phi$ is proportional to the symmetric multiplication $\left(u_{1}, u_{2}\right) \mapsto \lambda u_{1} u_{2}, \lambda \in \mathbb{R}$, because $S^{2} U=\mathfrak{h}$. This isomorphism is given by a choice of $\mathfrak{h}$-invariant area form $\omega$ on $U$. Let $p, q \in U$ be the canonical basis, $\omega(p, q)=1$. Then

$$
\Psi(p, \Phi(q, p))=\lambda \Psi(p, q p)=\lambda p \neq \Psi(q, \Phi(p, p))=\lambda \Psi\left(q, p^{2}\right)=-2 \lambda p
$$


unless $\lambda=0$. Thus the $\mathfrak{h}$ component vanishes and $[\mathfrak{r}, V] \subset \mathbb{C}$.

Therefore $[\mathfrak{r}, \mathfrak{m}] \subset \mathfrak{m}$ and $\mathfrak{r}$ acts by derivations.

This finishes the proof of Proposition 2.

4.2. Proof of Theorem 2. To find the derivations we can use the exact sequence

$$
0 \rightarrow Z(\mathfrak{m}) \longrightarrow \mathfrak{m} \stackrel{\text { ad }}{\longrightarrow} \mathfrak{d e r}(\mathfrak{m}) \longrightarrow H^{1}(\mathfrak{m}, \mathfrak{m}) \rightarrow 0,
$$

where $Z(\mathfrak{m})$ is the center of the Lie algebra $\mathfrak{m}$.

Consider at first the case $\mathfrak{h}=\mathfrak{s u}(2)$, representation $\mathfrak{a d}^{\mathbb{C}}$. In all four cases here $\mathfrak{m} \subset \mathfrak{g}$ is a Lie subalgebra.

In the first two cases A2.1, A2.2 it is semi-simple: $\mathfrak{s u}(2) \oplus \mathfrak{s u}(2)$ or $\mathfrak{s l}_{2}(\mathbb{C})$. By Whitehead lemma $H^{1}(\mathfrak{m}, \mathfrak{m})=0$, so all derivations are inner. Thus $\mathfrak{d} \mathfrak{e r}(\mathfrak{m})=\left\{\operatorname{ad}_{X}: X \in \mathfrak{m}\right\} \simeq \mathfrak{m}$ from $(3)$.

We claim that if $\operatorname{ad}_{X}$ commutes with $J$, then $\operatorname{ad}_{J X}$ does not. Elsewise

$$
N_{J}(X, Y)=\left[\operatorname{ad}_{J X}, J\right](Y)-J\left[\operatorname{ad}_{X}, J\right](Y)=0,
$$

and the Nijenhuis tensor is degenerate (even $\mathrm{DG}_{2}$ in terminology of $\left[\mathrm{K}_{1}\right]$ ). Therefore $\mathfrak{s y \mathfrak { m }}(J)_{o}$ is totally real in $\mathfrak{m}$ and so cannot have dimension $>3$. But dimension 3 is guaranteed since $\mathfrak{s y m}(J)_{o} \supset \mathfrak{h}$. Consequently $\mathfrak{s y \mathfrak { m }}(J)_{o}=\mathfrak{h}$ and $\mathfrak{s y \mathfrak { m }}(J)=\mathfrak{g}$.

Next case A2.3 is $\mathfrak{m}=\mathfrak{h} \ltimes \mathbb{R}^{3}$. Clearly $\mathfrak{s y m}(J)_{o} \subset \mathfrak{d e r}(\mathfrak{m})$ must preserve the radical $\mathbb{R}^{3}$. The operator $J_{r}=J-r \mathbf{1}$ is invariant, where $\mathbf{1}$ is the identity operator - see formula (1). Therefore the full isotropy also preserves the semisimple part $J_{r} \mathbb{R}^{3}=\mathfrak{h}$, and the action on $\mathfrak{h}$ induces the action on $\mathbb{R}^{3}$. Again any derivation on $\mathfrak{h}$ is internal by the Whitehead lemma and so $\operatorname{dim} \mathfrak{s y m}(J)_{o} \leq$ 3 implying $\mathfrak{s y \mathfrak { m }}(J)=\mathfrak{g}$.

The last case A2.4 is the graded nilpotent Lie algebra $\mathfrak{m}=\mathfrak{a}_{1} \oplus \mathfrak{a}_{2}$ with $\mathfrak{a}_{i} \simeq \mathbb{R}^{3}, J_{r}: \mathfrak{a}_{1} \rightarrow \mathfrak{a}_{2}$ and the bracket being given by $[\xi, \eta]=J_{r}(\xi \times \eta)$ for $\xi, \eta \in \mathfrak{a}_{1}$, the cross product $\times$ being the Lie bracket on $\mathbb{R}^{3}=\mathfrak{s u}(2)$. This relation shows that $\mathfrak{a}_{2}=[\mathfrak{m}, \mathfrak{m}]$ equipped with $\times$ product (so the bracket is $\left.\Lambda^{2} \mathfrak{a}_{2} \ni \xi \wedge \eta \mapsto\left[J_{r}^{-1} \xi, J_{r}^{-1} \eta\right] \in \mathfrak{a}_{2}\right)$ must be preserved by the derivations. Since this algebra $\left(\mathfrak{a}_{2}, \times\right)$ is isomorphic to $\mathfrak{s u}(2)$, we obtain $\operatorname{dim} \mathfrak{s y m}(J)_{o} \leq 3$ and $\mathfrak{s y m}(J)=\mathfrak{g}$ as before.

Finally there is a family of representations of $\mathfrak{h}$ of the type $V \oplus \mathbb{C}$. Only one of the cases A1.1, with $\mathfrak{m}$ being a Lie algebra, has non-degenerate $N_{J}$. In this case the space of derivations of $\mathfrak{m}$ commuting with $J$ is obtained by the straightforward computation with the case split according to parameters; these tedious computations are done in Maple. The result is the same as above.

The case $\mathfrak{h}=\mathfrak{s u}(1,1)$ is very similar to the considered $\mathfrak{s u}(2)$. The only difference is that in $V+\mathbb{C}$ representation there is one more case.

Now to complete the proof of Theorem 2, we have to consider the homogeneous structures of type II in Theorem 1 (when $\mathfrak{m}$ is not a Lie algebra: A1.4, A3.5). For such $M=G / H$ the Lie group $G$ is reductive, $\mathfrak{g}=\mathfrak{g}_{s s}+\mathfrak{z}$ with 
1-dimensional center $\mathfrak{z}$ and 8-dimensional semi-simple part $\mathfrak{g}_{s s}$, and the Lie algebra $\tilde{\mathfrak{g}}=\mathfrak{s} \mathfrak{y} \mathfrak{m}(J)$ contains $\mathfrak{g}$.

From the proof of Proposition 2 we know that $\tilde{\mathfrak{h}}=\mathfrak{h}+\mathfrak{r}$, where the semidirect summand $\mathfrak{r}$ is the radical in $\tilde{\mathfrak{h}}$ and $\operatorname{dim} \mathfrak{r} \leq 2$. This implies (by inspection of the Levi decomposition of $\tilde{\mathfrak{g}}$ ) that $\mathfrak{z}+\mathfrak{r} \subset \tilde{\mathfrak{g}}$ is a subalgebra, which is either semi-simple or the radical of $\tilde{\mathfrak{g}}$. In any case, because $\mathfrak{m}=\mathfrak{m}_{0}+\mathfrak{z}$ for $\mathfrak{m}_{0}=\mathfrak{m} \cap \mathfrak{g}_{s s} \subset \mathfrak{g}_{s s}$, we get $[\mathfrak{r}, \mathfrak{m}] \subset\left[\mathfrak{r}, \mathfrak{m}_{0}\right]+[\mathfrak{r}, \mathfrak{z}] \subset \mathfrak{r}+\mathfrak{z}$. Consequently $\pi \circ \mathfrak{a} \mathfrak{d}_{\mathfrak{r}}(\mathfrak{m}) \subset \mathfrak{z}$ for the projection $\pi: \tilde{\mathfrak{g}} \rightarrow \mathfrak{m}$ along $\tilde{\mathfrak{h}}$. Since $\pi \circ \mathfrak{a} \mathfrak{d}_{\mathfrak{r}} \subset \mathfrak{g l}(\mathfrak{m}, J)$ yields $J$-invariance of $\pi \circ \mathfrak{a d}_{\mathfrak{r}}(\mathfrak{m})$, we conclude that by dimensional reasons $\pi([\mathfrak{r}, \mathfrak{m}])=0$. Consequently the action of $\mathfrak{r}$ on $\mathfrak{m}$ is not effective, so $\mathfrak{r}=0$.

This finishes the proof of Theorem 2 .

\section{Almost Hermitian structures and their integrability}

The existence of a homogeneous almost Hermitian, almost symplectic or almost complex structure depends only on the isotropy representation, in contrast with the various integrability conditions (Kähler, etc.) for such structures which generally depend on the Lie algebra structure.

Pseudo-Riemannian metrics on the almost complex homogeneous manifold $M=G / H$ with the isotropy $\mathfrak{h}$-module $\mathfrak{m}$ and $\mathfrak{h}$-invariant complex structure $J$ on $\mathfrak{m}$ correspond to non-degenerate $\mathfrak{h}$-invariant quadratic forms $g \in S^{2} \mathfrak{m}^{*}$. Invariant almost Hermitian structures are elements of the set

$$
S_{J}^{2} \mathfrak{m}^{*}=\left\{g \in\left(S^{2} \mathfrak{m}^{*}\right)^{\mathfrak{h}}: g(J \xi, J \eta)=g(\xi, \eta), \operatorname{det}(g) \neq 0\right\} .
$$

Likewise invariant compatible almost symplectic structures are elements of the set

$$
\Lambda_{J}^{2} \mathfrak{m}^{*}=\left\{\omega \in\left(\Lambda^{2} \mathfrak{m}^{*}\right)^{\mathfrak{h}}: \omega(J \xi, J \eta)=\omega(\xi, \eta), \omega^{3} \neq 0\right\} .
$$

The Kähler form $\omega \in \Lambda_{J}^{2} \mathfrak{m}^{*}$ associated to $g \in S_{J}^{2} \mathfrak{m}^{*}$ is defined by $\omega(\xi, \eta)=$ $g(J \xi, \eta)$. This formula makes a bijective correspondence $S_{J}^{2} \mathfrak{m}^{*} \simeq \Lambda_{J}^{2} \mathfrak{m}^{*}$.

Note that two invariant almost Hermitian metrics $g, \tilde{g}$ define a symmetric (with respect to both $g$ and $\tilde{g}$ ) invertible operator $A: \mathfrak{m} \rightarrow \mathfrak{m}$ by $\tilde{g}(\xi, \eta)=$ $g(A \xi, \eta)$. Since both sides are $\mathfrak{h}$-invariant, this $A$ commutes with $\mathfrak{h}$. Moreover the compatibility condition implies that $A$ is complex linear, $[A, J]=0$. Thus the operator $A$ belongs to the complex endomorphism $\operatorname{ring} \operatorname{End}_{\mathfrak{h}}(\mathfrak{m}, J)$.

5.1. Classification of almost Hermitian structures. Let us list all invariant almost Hermitian structures according to the types of $\mathfrak{h}$-modules as in Theorem 3 (we'll omit the word "almost" for the metric).

Case 1: $\mathfrak{h}=\mathfrak{s u}(2), \mathfrak{m}=V \oplus \mathbb{C}$, where $V \simeq \mathbb{H}$. There are Hermitian metrics $g_{1}$ on $V, g_{2}$ on $\mathbb{C}$. Since $\operatorname{End}_{\mathfrak{h}}(\mathfrak{m}, J)=\mathbb{C} \oplus \mathbb{C}$ (acting submodulewise) and the only symmetric endomorphism are $A \in \mathbb{R} \oplus \mathbb{R}$, the general invariant compatible metric on $\mathfrak{m}$ is $g=a g_{1}+b g_{2}$. The signature of $g$ is any even $(2 k, 6-2 k)$ depending on $a, b \neq 0$.

The almost-symplectic form on $\mathbb{C}$ component is unique up to scaling (which we fix). Since the endomorphism ring of the first component is $\operatorname{End}_{\mathfrak{h}}(V)=\mathbb{H}$ and only imaginary quaternions are skew-symmetric, we conclude $\omega(\xi, \eta)=$ 
$g_{1}\left(\xi_{1} q, \eta_{1}\right)+g_{2}\left(i \xi_{2}, \eta_{2}\right)$ for some $q \in \operatorname{Im}(\mathbb{H}) \backslash 0$, where $\xi=\xi_{1}+\xi_{2}, \eta=\eta_{1}+\eta_{2} \in$ $V \oplus \mathbb{C}$.

Case 2: $\mathfrak{h}=\mathfrak{s u}(2), \mathfrak{m}=\mathfrak{a d}^{\mathbb{C}}$. The operator $J$ induces the equivariant splitting $\mathfrak{m}=\mathfrak{a} \mathfrak{d} \oplus J \mathfrak{a} \mathfrak{d}$. The Riemannian metric $g$, which is the direct sum of the Killing forms on each summand is compatible. Since $\mathfrak{m}$ is a complex irreducible representation, $\operatorname{End}_{\mathfrak{h}}(\mathfrak{m}, J)=\mathbb{C}$. Then $g(A \xi, \eta)$ for $A \in$ $\operatorname{End}_{\mathfrak{h}}(\mathfrak{m}, J)$ is non-symmetric unless $A \in \mathbb{R}$, and so the invariant compatible metric is unique up to scaling (but $g$ depends on $J$ ).

Decomposing $\mathfrak{h}$-modules $\Lambda^{2} \mathfrak{m}=3 \Lambda^{2} \mathfrak{a} \mathfrak{d} \oplus S^{2} \mathfrak{a} \mathfrak{d}=3 \mathfrak{a} \mathfrak{d} \oplus W^{5} \oplus \mathbb{R}^{1}$, where $W^{5}$ is irreducible and $\mathbb{R}^{1}$ trivial, we conclude that the only almost symplectic form comes from the compatible metric.

Case 3: $\mathfrak{h}=\mathfrak{s u}(1,1), \mathfrak{m}=V \oplus \mathbb{C}$. Here $V=U^{\mathbb{C}}$ for the standard $\mathfrak{s l}_{2}$ representation $U$. This case is similar to Case 1 , and the general invariant metric is again $g=a g_{1}+b g_{2}, a, b \neq 0$. Now however the metric $g_{1}$ is of split signature, $g_{2}$ is Riemannian, and so $g$ has type $(4,2)$ (or $(2,4)$, but we will not distinguish the opposite signatures).

Since $\Lambda^{2} V=\mathfrak{a} \mathfrak{d} \oplus \mathbb{R}^{3}$, the space of invariant 2-forms is 3-dimensional. Indeed, $\operatorname{End}_{\mathfrak{h}}(V)=\mathfrak{g l}_{2}$, and so a general almost symplectic form is $\omega(\xi, \eta)=$ $g_{1}\left(A \xi_{1}, \eta_{1}\right)+g_{2}(i \xi, \eta), A \in \mathrm{GL}_{2}$. We can write this via split quaternions $\mathbb{H}_{s}$ (isomorphic to $\mathfrak{g l}_{2}$ as algebra) so: $\omega(\xi, \eta)=g_{1}\left(\xi_{1} q, \eta_{1}\right)+g_{2}\left(i \xi_{2}, \eta_{2}\right), q \in$ $\operatorname{Im}\left(\mathbb{H}_{s}\right), q^{2} \neq 0$.

Case 4: $\mathfrak{h}=\mathfrak{s u}(1,1), \mathfrak{m}=\mathfrak{a d}^{\mathbb{C}}$. This is similar to Case 2: the (invariant) almost symplectic structure $\omega$ is unique up to scale; it is $J$-independent and is $J$-compatible for every $J \in \operatorname{End}_{\mathfrak{h}}(\mathfrak{m}), J^{2}=\mathbf{- 1}$. The Hermitian metric $g=-i_{J} \omega$ depends on $J$ and has signature $(4,2)$.

Case 5: $\mathfrak{h}=\mathfrak{s l}_{2}(\mathbb{C}), \mathfrak{m}=V+\mathbb{C}$. Since $\mathfrak{s l}_{2}(\mathbb{C})$ contains the subalgebras $\mathfrak{s u}(2)$ and $\mathfrak{s u}(1,1)$, the metric on $V$ component must be invariant under both of them, and no such metric exists.

Consider the 2-form $\omega_{1}(x, y)=g_{1}(x q, y)$ on $V$ invariant with respect to $\mathfrak{s u}(2), q \in \operatorname{Im}(\mathbb{H})$, see Case 1 . Since $\mathfrak{s u}(1,1)$ can be identified with $i \mathfrak{s u}(2)$ inside $\mathfrak{s l}_{2}(\mathbb{C})$, we compute that $\omega_{1}$ is $\mathfrak{s u}(1,1)$-invariant iff $q \in \operatorname{Im}(\mathbb{H}) \cap i \operatorname{Im}(\mathbb{H})$, i.e. $q \perp i$. On the $\mathbb{C}$ factor the invariant symplectic form is unique. Thus the space of invariant almost symplectic structures on $\mathfrak{m}$ is given by 2 parameters up to scale: $\omega=\omega_{1}+\omega_{2}$.

Case 6: $\mathfrak{h}=\mathfrak{s l}_{2}(\mathbb{C}), \mathfrak{m}=\mathfrak{a}$. The Killing form $K$ provides an invariant metric on $\mathfrak{a} \mathfrak{d}$, but it is not Hermitian as $K(J \xi, J \eta)=-K(\xi, \eta)$. Since any other metric or 2-form must be related to $K$ by an operator $A \in \operatorname{End}_{\mathfrak{h}}(\mathfrak{m})=$ $\mathbb{C}$, no compatible metric and no almost-symplectic form exists. Instead we have two invariant anti-compatible metrics $K(\xi, \eta)$ and $K(J \xi, \eta)$. The almost complex structure $J=i$ on $\mathfrak{m}$, and hence on the corresponding homogeneous manifold $M=\mathrm{SL}_{2}(\mathbb{C}) \times \mathrm{SL}_{2}(\mathbb{C}) / \mathrm{SL}_{2}(\mathbb{C})_{\text {diag }} \simeq \mathrm{SL}_{2}(\mathbb{C})$, the structure $J$ is integrable.

Case 7: $\mathfrak{h}=\mathfrak{s u}(3), \mathfrak{m}=V$. By definition we have an invariant Hermitian metric $g$ of signature $(6,0)$ on $\mathfrak{m}$. The endomorphism ring is $\mathbb{C}$, but $g(A \xi, \eta)$ 
is not symmetric unless $A \in \mathbb{R}$, so $g$ is unique up to scaling. The almost symplectic form is also unique and compatible.

The corresponding homogeneous manifold is $\mathbb{S}^{6}$, and $J$ is the unique invariant almost complex structure. Known as the Calabi structure, it is well studied. In particular, the triple $(g, J, \omega)$ is strongly nearly Kähler (SNK) and the Hermitian metric $g$ is 3-symmetric and Einstein.

Case 8: $\mathfrak{h}=\mathfrak{s u}(2,1), \mathfrak{m}=V$. By definition we have an invariant pseudoHermitian metric $g$ of signature $(4,2)$ on $\mathfrak{m}$. The endomorphism ring is $\mathbb{C}$, but $g(A \xi, \eta)$ is not symmetric unless $A \in \mathbb{R}$, so again the pseudo-Riemannian metric $g$ and the almost symplectic form $\omega$ are both unique (up to scaling) and compatible.

The corresponding homogeneous manifold is $\mathbb{S}^{2,4} \simeq \mathbb{S}^{2} \times \mathbb{R}^{4}$, and $J$ is the unique invariant almost complex structure. It is not integrable, has nondegenerate Nijenhuis tensor, and is the split analog of the Calabi structure. The triple $(g, J, \omega)$ is strongly nearly pseudo-Kähler and the Hermitian metric $g$ is Einstein.

5.2. Kähler and nearly Kähler structures. Examining the list of all our homogeneous structures we conclude that the only Kähler metrics are the cases A1.1, A3.1 and A1.3, A3.4 of Tables from the Appendix (we pair the similar cases). Even though the groups on which the structures live are solvable (the topology is rather simple), the metric properties are non-trivial. We summarize the results.

Theorem 5. The only (pseudo-)Kähler homogeneous 6D manifolds with semi-simple (nontrivial) isotropy are quotients $M=G / H$ with $H=S U(2)$ or $H=S U(1,1)$ with reducible isotropy representation $\mathfrak{m}=V+\mathbb{C}$. As an $H$-module $V=\mathbb{H}$ or resp. $V=\mathbb{H}_{s}$.

The corresponding reductive complement $\mathfrak{m}$ is a Lie algebra, so $M$ is a (quotient of) Lie group with the Lie algebra given by the following relations (two cases). Below $\alpha, r \in \mathbb{R}, \varepsilon \in\{0,1\}$ are the parameters, $\alpha \neq 0$, and the vectors $x, y \in V$, e, $i \mathrm{e} \in \mathbb{C}$.

1) $\mathfrak{m}: \quad[x, y]=\alpha \operatorname{Re}(x i \bar{y}) \mathrm{e},[x, i \mathrm{e}]=x\left(\frac{1}{2}+r i\right),[\mathrm{e}, i \mathrm{e}]=\mathrm{e}$.

Thus $\mathfrak{m}^{6}$ is 1-dimensional "right5 extension" of the 5D Heisenberg algebra. The symplectic form is $\omega=\alpha \omega_{V}+\omega_{\mathbb{C}}, \omega_{V}(x, y)=\operatorname{Re}(x i \bar{y}), \omega_{\mathbb{C}}(\mathrm{e}, i \mathrm{e})=1$. The Hermitian metric is $g(\xi, \eta)=\omega(\xi, J \eta)$ with $J(x, \mathrm{e})=(x i, i \mathrm{e})$ in the decomposition $V+\mathbb{C}$, with signature $(6,0)$ for $\mathfrak{h}=\mathfrak{s u}(2)$ and $\alpha>0$ and signature $(4,2)$ else. Moreover $g$ is Einstein with the cosmological constant -4 , and is not conformally flat.

2) $\mathfrak{m}:$

$$
[x, i \mathrm{e}]=r x i, \quad[\mathrm{e}, i \mathrm{e}]=\varepsilon \mathrm{e}
$$

\footnotetext{
${ }^{5}$ This means extension by derivations; terminology comes from Fuks $[\mathrm{F}$, and is opposed to left=central extensions. For a Lie algebra $\mathfrak{g}$ its "right extensions" are enumerated by the cohomology group $H^{1}(\mathfrak{g}, \mathfrak{g})$ and "left extensions" by $H^{2}(\mathfrak{g})$.
} 
Thus $\mathfrak{m}^{6}$ is a 1-dimensional "right extension" of the 5D Abelian algebra. The symplectic form is $\omega=\omega_{V}+c \omega_{\mathbb{C}}$. The Hermitian metric is again $g(\xi, \eta)=$ $\omega(\xi, J \eta)$ with $J(x, \mathrm{e})=(x i, i \mathrm{e})$. It has signature $(6,0)$ for $\mathfrak{h}=\mathfrak{s u}(2), c>0$ and signature $(4,2)$ else. The metric is not Einstein or conformally flat unless $\varepsilon=0$, when $g$ is flat.

It is also interesting to study when $(M, g, J, \omega)$ is strongly nearly Kähler (SNK), meaning that for the Levi-Civita connection $\nabla$ the tensor $\nabla \omega$ is (nonzero) totally skew symmetric $\left(\nabla \omega=\frac{1}{3} d \omega \neq 0\right)$. It is a restrictive condition. For instance, the Nijenhuis tensor $N_{J}$ is non-degenerate and the geometry is constrained by the 'splitting principle' of P.-A. Nagy [Na]. Homogeneous SNK structures were classified by J.-B. Butruilles [Bu]. The classification up to a covering is:

- $\mathbb{S}^{6}=G_{2}^{c} / S U(3)$,

- $\mathbb{S}^{3} \times \mathbb{S}^{3}=S U(2) \times S U(2) \times S U(2) / S U(2)_{\operatorname{diag}}$

- $\mathbb{C} P^{3}=S U(4) /(S U(3) \times U(1))=S p(4) /(S U(2) \times U(1))$,

- the flag variety $\mathbb{F}(1,2)=S U(3) /(U(1) \times U(1))$.

The first two belong to our list (the invariant structure $J$ on $\mathbb{S}^{3} \times \mathbb{S}^{3}$ corresponds to the case A2.1 from the Tables with parameters $(r, t)= \pm\left(\frac{1}{\sqrt{3}}, \frac{2}{\sqrt{3}}\right)$, so it has more symmetry than observed in $[\mathrm{Bu}]$ ), while the last two do not (as they have reductive and Abelian isotropy respectively, and can be detected by a refinement of our calculation).

Homogeneous pseudo-SNK of signature $(2,4)$ (this is given by the same condition: $\nabla \omega$ nonzero totally skew symmetric) with semi-simple isotropy can be extracted from our classification 6 :

- $\mathbb{S}^{2,4}=G_{2}^{*} / S U(1,2)$,

- $S L(2) \times S L(2)=S U(2,1) \times S U(2,1) \times S U(2,1) / S U(2,1)_{\operatorname{diag}}$ (the invariant structure $J$ on this $M^{6}$ corresponds to the case A4.1 of the Tables with parameters $\left.(r, t)= \pm\left(\frac{1}{\sqrt{3}}, \frac{2}{\sqrt{3}}\right)\right)$

- the left-invariant structure on the (solvable) Lie group with Lie algebra from the case A3.2 of the Tables with parameters (after rescaling $\omega)$ equal to $r=-\frac{3}{2 t}, \epsilon=+1, \alpha=0, p=t(i+j) \in \mathbb{H}_{s}, u=-\frac{1}{2} k \in \mathbb{H}_{s}$, $q=i$ and $b=\frac{1}{2} t i \in \mathbb{H}_{s}$.

Remark 3. Since the latter homogeneous pseudo-SNK structure does not have an SNK analog (the first two have obviously the same complexification as the first two entries of Butruilles' list), let us write the structures explicitly.

\footnotetext{
${ }^{6}$ There are obvious pseudo-SNK analogs of signature $(2,4)$ of the last two entries in Bitruilles' list, but we present here only the spaces $G / H$ with semi-simple $H$.
} 
The structure equations of the Lie algebra $\mathfrak{m}$ are $(t \neq 0)$ :

$$
\begin{gathered}
{\left[e_{1}, e_{2}\right]=t e_{5},\left[e_{1}, e_{3}\right]=-t e_{5},\left[e_{2}, e_{4}\right]=-t e_{5},\left[e_{3}, e_{4}\right]=t e_{5},} \\
{\left[e_{1}, e_{5}\right]=-\frac{3}{2}\left(e_{2}+e_{3}\right),\left[e_{2}, e_{5}\right]=\frac{3}{2}\left(e_{1}-e_{4}\right),\left[e_{3}, e_{5}\right]=\frac{3}{2}\left(e_{4}-e_{1}\right),} \\
{\left[e_{4}, e_{5}\right]=-\frac{3}{2}\left(e_{2}+e_{3}\right),\left[e_{1}, e_{6}\right]=e_{1}-\frac{1}{2} e_{4},\left[e_{2}, e_{6}\right]=e_{2}+\frac{1}{2} e_{3},} \\
{\left[e_{3}, e_{6}\right]=e_{3}+\frac{1}{2} e_{2},\left[e_{4}, e_{6}\right]=e_{4}-\frac{1}{2} e_{1},\left[e_{5}, e_{6}\right]=e_{5}}
\end{gathered}
$$

and the almost complex structure $J$ and the metric $g$ are given by the formulae (we denote by $\theta_{i}$ the dual basis of $\mathfrak{m}^{*}$ )

$$
\begin{gathered}
J=\left(e_{2} \otimes \theta_{1}-e_{1} \otimes \theta_{2}+e_{3} \otimes \theta_{4}-e_{4} \otimes \theta_{3}\right)+\left(e_{6} \otimes \theta_{5}-e_{5} \otimes \theta_{6}\right), \\
g=\frac{1}{2} t\left(\theta_{1}^{2}+\theta_{2}^{2}-\theta_{3}^{2}-\theta_{4}^{2}\right)+\theta_{5}^{2}+\theta_{6}^{2}
\end{gathered}
$$

Notice that though this (1-parametric) structure lives on a Lie group $M^{6}$ (or its finite quotient), it has the symmetry group of dimension 9 and so can be represented on the homogeneous space $G^{9} / H^{3} \simeq M^{6}$.

5.3. SKT and Gray-Hervella classes. In the next sections we will also study the strong Kähler with torsion (SKT) property $\partial \bar{\partial} \omega=0$ (in addition to $N_{J}=0$ ) important in generalized Kähler geometry and supersymmetric nonlinear sigma models. The named property is equivalent to

$$
d_{J}^{2} \omega=0, \quad d_{J}=d \circ J
$$

(where $J \circ \sigma=\sigma(J \cdot, J \cdot, .$.$) ), and we shall study generalizations when d_{J}^{k} \omega=0$ for larger $k$ (and $J$ not necessarily integrable). For instance, the standard almost Hermitian structure $(g, J, \omega)$ on $\mathbb{S}^{6}$ is not SKT and $d_{J}^{3} \omega \neq 0$, but the derived top form vanishes: $d_{J}^{4} \omega=0$.

There are many structures $J$ of type III, which are not SKT, but satisfy the condition $d_{J}^{3} \omega=0$. The only occasions of SKT are these:

Theorem 6. The only homogeneous Hermitian manifolds $M^{6}$ with semisimple isotropy, which satisfy the SKT property but do not belong to either Kähler or pseudo-Kähler class, are equivalent to the following.

1) The structure of case A1.2 with parameters $q=\cos \theta \cdot i+\sin \theta \cdot j$, $p= \pm \sqrt{3 \sin ^{2} \theta-1} \cdot q+\sin \theta \cdot k$. The Lie algebra $\mathfrak{m}$ is the central extension

$$
0 \rightarrow \mathbb{R}^{2} \longrightarrow \mathfrak{m} \longrightarrow \mathbb{R}^{4} \rightarrow 0,
$$

whence the homogeneous space is $M=G / H$ is an $\mathbb{R}^{2}$-bundle over $\mathbb{R}^{4}$.

2) The structure of case A1.3 with parameters $q=i, \alpha=0, \beta=-\frac{1}{2}, \epsilon=1$ or of case A3.4 with the same parameters and in addition $p=0, u=\lambda i$. The Lie algebra $\mathfrak{m}$ is the "right" extension

$$
0 \rightarrow \mathbb{R}^{4} \longrightarrow \mathfrak{m} \longrightarrow \mathfrak{s}_{2} \rightarrow 0
$$

where $\mathfrak{s}_{2}=\operatorname{Lie}\left(S_{2}\right)$ is the solvable non-abelian $2 D$ Lie algebra of the Lie group $S_{2}$, represented via rank 1 homomorphism $S_{2} \rightarrow \mathbb{C}^{*} \stackrel{\text { diag }}{\hookrightarrow} \mathrm{GL}_{2}(\mathbb{C}) \subset \mathrm{GL}_{4}(\mathbb{R})$ (in both cases $\mathbb{R}^{4}=V=\mathbb{H}$ or $\mathbb{H}_{s}$ is equipped with the complex structure $i$ ). The homogeneous space is $M=G / H \simeq \mathbb{R}^{4} \rtimes S_{2}$. 
3) The structure of case A3.4 with parameters $q=i, \alpha=0, \epsilon=1$, $p=\frac{1}{2}(i+j), u=-\frac{1}{2} k$ and $\beta=-1$ or $\beta=\frac{1}{2}$. The Lie algebra $\mathfrak{m}$ is the "right" extension given by the same sequence as in 2), but now the homomorphism $\varphi: S_{2} \rightarrow \mathrm{GL}_{2}(\mathbb{C}) \subset \mathrm{GL}_{4}(\mathbb{R})$ has rank 2: $\varphi(\mathrm{e})=R_{\frac{1}{2}(i+j)}, \varphi(i \mathrm{e})=R_{\beta-\frac{1}{2} k}$, where $R_{h}$ is the right multiplication by the split-quaternion $h$. Again the homogeneous space is $M=G / H \simeq \mathbb{R}^{4} \rtimes S_{2}$.

It is also interesting which Gray-Hervella (GH) classes of almost Hermitian manifolds are realizable within our homogeneous 6D manifolds with semisimple isotropy (in our classification only Tables A1 and A2 correspond to almost Hermitian manifolds, so in what follows we refer only to them). In the work [GH] 16 classes of such manifolds were encoded by the set $\mathcal{P}(\{1,2,3,4\})$ of all subsets of the 4-point set.

The class corresponding to the empty set $\emptyset$ is the Kähler class $\mathcal{K}$. The class $\mathcal{W}$ corresponding to the whole set $\{1,2,3,4\}$ consists of all almost Hermitian manifolds. The basic GH-classes $\mathcal{W}_{i}, 1 \leq i \leq 4$, correspond to 1-point subsets. The other classes correspond to the direct sum of representations $W_{i}$ of the basic classes (equivalently the basic modules can be taken $W \ominus W_{i}$, the other modules being the intersections of these; the non-trivial GH-classes are then given by the union of the conditions determining $\sum_{j \neq i} \mathcal{W}_{j}$.

In order to represent the general class $\mathcal{W}$ by a disjoint union, we modify the Gray-Hervella classes to the classes $\tilde{\mathcal{W}}_{\sigma}, \sigma \in \mathcal{P}(\{1,2,3,4\})$. Namely, $\tilde{\mathcal{W}}_{\sigma}$ consists of all elements of $\sum_{i \in \sigma} \mathcal{W}_{i}$, which do not belong to $\tilde{\mathcal{W}}_{\tau}$ with $\tau \varsubsetneqq \sigma$. This definition is inductive starting from $\tilde{\mathcal{W}}_{\emptyset}=\mathcal{K}$. For instance, $\tilde{\mathcal{W}}_{i}=\mathcal{W}_{i} \backslash \mathcal{K}$, $\tilde{\mathcal{W}}_{i, j}=\left(\mathcal{W}_{i} \oplus \mathcal{W}_{j}\right) \backslash\left(\mathcal{W}_{i} \cup \mathcal{W}_{j}\right)$ for $i \neq j$ etc.

We already discussed and classified the Kähler $\mathcal{K}$ and nearly Kähler $\mathcal{W}_{1}=$ $\mathcal{N K}$ classes, which also gives the description of strictly nearly Kähler class $\tilde{\mathcal{W}}_{1}$. Inspection of the tables rules out the class $\mathcal{W}_{2}=\mathcal{A K}$ of almost Kähler manifolds with the exception of the Kähler structures (observe from the Tables A1-A2 that whenever $d \omega=0$, then also $N_{J}=0$, so the structure belongs to the Kähler class $\mathcal{K}$ ). The class $\mathcal{W}_{3}=\mathcal{H} \cap \mathcal{S K}$ of special Hermitian manifolds is realized only (again with the exception of the Kähler class $\mathcal{K}$ ) by the Tables A1.2 (several parametric cases) and A2.2 $(r=0, t= \pm 1)$; the latter is $M=S L_{2}(\mathbb{C})$ with the standard complex structure. The class $\tilde{\mathcal{W}}_{4}$ (containing locally conformally Kähler but nohn-Kähler manifolds) is realized only by the Table A1.4 $(q= \pm i)$, in particular for $\varepsilon=-1$ we obtain the Calabi-Eckmann structure on $\mathbb{S}^{1} \times \mathbb{S}^{5}$. For the other classes we have:

Theorem 7. The GH-classes, realized as homogeneous almost Hermitian manifolds $M^{6}$ with semi-simple isotropy, are precisely the following: $\mathcal{K}, \tilde{\mathcal{W}}_{1}$, $\tilde{\mathcal{W}}_{3}, \tilde{\mathcal{W}}_{4}, \tilde{\mathcal{W}}_{1,2}, \tilde{\mathcal{W}}_{1,3}, \tilde{\mathcal{W}}_{2,3}, \tilde{\mathcal{W}}_{3,4}, \tilde{\mathcal{W}}_{1,2,3}, \tilde{\mathcal{W}}_{1,2,4}, \tilde{\mathcal{W}}_{1,3,4}, \tilde{\mathcal{W}}_{2,3,4}, \tilde{\mathcal{W}}=\tilde{\mathcal{W}}_{1,2,3,4}$

Thus the non-realizable (via our models) GH-classes are $\tilde{\mathcal{W}}_{2}, \tilde{\mathcal{W}}_{1,4}, \tilde{\mathcal{W}}_{2,4}$.

Proof. The proof is the direct calculation (in this algebraic computation we used Maple). Let us indicate, which sub-classes in Tables A1 and A2 realize the GH-classes (omitting the precise values of the parameters): 

A1.1: $\mathcal{K}, \tilde{\mathcal{W}}_{3,4}, \tilde{\mathcal{W}}_{1,2,3}, \tilde{\mathcal{W}}$
A1.2: $\tilde{\mathcal{W}}_{3}, \tilde{\mathcal{W}}_{1,2}, \tilde{\mathcal{W}}_{3,4}, \tilde{\mathcal{W}}_{1,2,3}, \tilde{\mathcal{W}}$
A2.1: $\tilde{\mathcal{W}}_{1}, \tilde{\mathcal{W}}_{1,3}$
A1.3: $\mathcal{K}, \tilde{\mathcal{W}}_{3,4}, \tilde{\mathcal{W}}_{1,2,3}, \tilde{\mathcal{W}}$
A2.2: $\tilde{\mathcal{W}}_{3}, \tilde{\mathcal{W}}_{1,3}$.
A1.4: $\tilde{\mathcal{W}}_{4}, \tilde{\mathcal{W}}_{1,2}, \tilde{\mathcal{W}}_{1,3}, \tilde{\mathcal{W}}_{2,3}, \tilde{\mathcal{W}}_{i, j, k}(i<j<k), \tilde{\mathcal{W}}$.
A2.3: $\tilde{\mathcal{W}}_{1,3}$.
A2.4: $\tilde{\mathcal{W}}_{1,3}$.

Notice that for Table A2 the structures are never in the general $\tilde{\mathcal{W}}$ class because they always satisfy the condition $\delta \omega=0$ of $\mathcal{W}_{1} \oplus \mathcal{W}_{2} \oplus \mathcal{W}_{3}$ by the $\mathfrak{h}$-equivariance of the divergence and the module type, but in fact they all satisfy the stronger condition of $\mathcal{W}_{1} \oplus \mathcal{W}_{3}$.

Remark 4. The cocalibrated structures of Hervella-Vidal type $\mathcal{G}_{1}=\mathcal{W}_{1} \oplus$ $\mathcal{W}_{3} \oplus \mathcal{W}_{4}$ are those admitting a Hermitian connection with totally skewsymmetric torsion studied in [AFS, S] ]. Our computation confirms the results of these papers about the GH-type of such structures with the parallel torsion.

\section{InVESTigation OF HOMOgEnEOUS MODELS OF TYPE II}

The $G_{2}$-invariant almost complex structures on $\mathbb{S}^{6}$ and $\mathbb{S}^{2,4}$ (type I) are well-studied $\left[\mathrm{E}, \mathrm{G}, \mathrm{Ka}, \mathrm{K}_{2}\right.$, type III structures are described in Appendix - the corresponding manifolds have simple topology. In this section we describe the almost complex models of type II (some examples of these appeared in [S]).

Notice that by Section 5 none of these possesses a Kähler structure invariant with respect to the corresponding group $G$ of dimension 9 . This does not mean that they do not have Kähler structures at all. We shall describe them topologically and see that in some cases such a structure exists, however it is not $G$-invariant.

6.1. Homogeneous models of type $\mathbf{I I}_{1}$. Structures of type $\mathrm{II}_{1}$ have $\mathfrak{m}=$ $V+\mathbb{C}$ as $\mathfrak{h}$-representation. Interpreting $\mathfrak{h}=\mathfrak{s u}(2)$ as imaginary quaternions we can identify $V$ with the space of quaternions $\mathbb{H}$ (as the module, not algebra). In particular, the set of $\mathfrak{h}$-invariant complex structures is the standard unit sphere $\mathbb{S}^{2}=\{q \in \operatorname{Im}(\mathbb{H}): q \cdot q=-1\}$.

Thus the set of $\mathfrak{h}$-invariant (almost) complex structures on (representation) $\mathfrak{m}$ is $\mathcal{J}_{e}=\mathbb{S}^{2} \times\{1\} \simeq \mathbb{S}^{2}$.

$\mathbf{I I}_{1}^{a}$. The Lie group $S U(3)$ acts transitively on $\mathbb{S}^{5}$ with the stabilizer $S U(2)$. Therefore we have the following diffeomorphism:

$$
M=U(3) / S U(2)=U(1) \times S U(3) / S U(2) \simeq \mathbb{S}^{1} \times \mathbb{S}^{5} .
$$

The complex structure $J$ on this manifold is obtained from Hopf fibration $\mathbb{S}^{5} \rightarrow \mathbb{C} P^{2}$ with the fiber $\mathbb{S}^{1}$ and the standard connection $H \subset T \mathbb{S}^{5}$, so that $M$ is the $\mathbb{T}^{2}$ fibration over $\mathbb{C} P^{2}$.

This is the well-known Calabi-Eckmann complex structure $J_{0}$ : at every point it is the sum of the standard complex structure on the connection (flat CR-structure) and a complex structure on $\mathbb{T}^{2}=\mathbb{S}^{1} \times \mathbb{S}^{1}$ (given by 2 parameters, which disappear if we pass to the universal cover). Clearly, $M$ is not symplectic and so is not Kähler. 
There is however a 2-parameter family of deformations of this structure to almost complex structure on the universal cover $\tilde{M}=\mathbb{R}^{1} \times \mathbb{S}^{5}$. Indeed, this is given by the construction of $(\mathfrak{g}, \mathfrak{h}, \mathfrak{m}, J)$, see Tables in the Appendix. To describe $J$ fix a point, say $a=(0,0,1) \in \mathbb{S}^{5}$. The CR-hyperplane $\mathbb{C}^{2} \simeq H_{a} \subset$ $T_{a} \mathbb{S}^{5}$ as $\mathfrak{h}$-representation is isomorphic to $\mathbb{H}$, and so the space of invariant structures is $\mathbb{S}^{2}$. We translate this to any other point $b \in \mathbb{S}^{5}$ by an element of $S U(3)$ and obtain an invariant CR-structure $J^{\prime}$ on the connection $H$ (in fact, we have the trivial $\mathbb{S}^{2}$-bundle of almost complex structures on the connection $H$ over $\mathbb{S}^{5}$ ). This is extended to $J=J^{\prime}+J^{\prime \prime}$ by complementing $J^{\prime}$ with (any) complex shift invariant structure $J^{\prime \prime}$ on the cylinder $\mathbb{R}^{1} \times \mathbb{S}^{1} \simeq \mathbb{C} / \mathbb{Z}$. The space of obtained structures is $\mathcal{J}_{e} \simeq \mathbb{S}^{2}$.

Since normalization of the Lie algebra structure on $\mathfrak{g}$ involves complex multiplication $J_{0}=i$ on $\mathfrak{m}$, there are two preferred complex structures $\pm J_{0}$ on this sphere. Moving along the fiber of the Hopf fibration $\mathbb{S}^{5} \rightarrow \mathbb{C} P^{2}$ rotates the sphere $\mathbb{S}^{2}$ along the axis through these antipodal points (the differential of this maps $\left.\operatorname{Im}(\mathbb{H}) \ni q \mapsto[q, i] \in T \mathbb{S}^{2}\right)$. Only $\pm J_{0}$ are (integrable) complex structures; for all other choices of $J$, the Nijenhuis tensor $N_{J}$ is nondegenerate.

When we compactify $M$ to $\mathbb{S}^{1} \times \mathbb{S}^{5}$, we get two more parameters coming from the torus $\mathbb{T}^{2} \subset U(3) / S U(2)$ (quotient of the cylinder), as the space of complex structures on it is given by the fundamental domain $\Sigma^{2}=\{|z| \geq$ $\left.1,|\operatorname{Re}(z)| \leq \frac{1}{2}, \operatorname{Im}(z)>0\right\} \subset \mathbb{C}$. Thus the space of parameters of $U(3)$ invariant complex structures on this compact $M^{6}$ is $\mathcal{J}_{+}=\mathcal{J}_{e} \times \Sigma^{2}$. The latter deformation does not change integrability: $N_{J}$ depends only on the first factor, and so is as described above.

$\mathbf{I I}_{1}^{b}$. Similarly, the Lie group $S U(2,1)$ acts transitively on the pseudosphere $N^{5}=\left\{z \in \mathbb{C}^{3}:\left|z_{1}\right|^{2}+\left|z_{2}\right|^{2}-\left|z_{3}\right|^{2}=-1\right\}$ with the stabilizer $S U(2)$ (inducing the action on the unit ball $B^{4} \simeq \mathbb{P} N^{5}$ with the stabilizer $U(2)$, important for the canonical/flat CR-structure on $\left.\mathbb{S}^{3}=\partial B^{4}\right)$. Since $N^{5} \simeq$ $\mathbb{S}^{1} \times B^{4}$, we obtain the following diffeomorphism:

$$
M=U(2,1) / S U(2) \simeq U(1) \times S U(2,1) / S U(2) \simeq \mathbb{T}^{2} \times B^{4} .
$$

The invariant complex structure $J$ on this $M$, as well as on the universal cover $\tilde{M}=\mathbb{C} \times B^{4}$, is however not the obvious one, since $(M, J, \omega)$ is not Kähler: the structure $J^{\prime}$ on the factor $B^{4}$ has to be $S U(2,1)$-invariant.

Again the space of moduli of all $S U(2)$-invariant structures $J$ on $\tilde{M}$ is $\mathcal{J}_{e} \simeq \mathbb{S}^{2}$ and on $M$ it is $\mathcal{J}_{+}=\mathcal{J}_{e} \times \Sigma^{2}$. Indeed, at every point $a \in N^{5}$ the natural contact space $H_{a} \subset T_{a} N^{5}$ is isomorphic to $\mathbb{H}$ as $\mathfrak{h}$-module, and the space of invariant complex structures on $H$ is the trivial $\mathbb{S}^{2}$-bundle over $N^{5}$. This gives the complex structure $J^{\prime}$, and the shift invariant complex structure $J^{\prime \prime}$ on the factor $\mathbb{T}^{2}$ resp. $\mathbb{C}$ yields the structure $J=J^{\prime}+J^{\prime \prime}$ on $M$ resp. $\tilde{M}$. The integrable structures among these $J$ are $J_{0}= \pm i$ only, for others the tensor $N_{J}$ is non-degenerate.

Concerning the integrability properties for the structures from $\mathcal{J}_{e}$ the following statement describes the generalized SKT property. 
Proposition 3. For the compatible invariant almost symplectic form $\omega$ and the operator $d_{J}=d \circ J$ we have: $d_{J}^{2} \omega \neq 0$ always; $d_{J}^{3} \omega \neq 0$ unless $J=J_{0}= \pm i$ or $J \perp J_{0} \Leftrightarrow J=\cos t \cdot j+\sin t \cdot k, t \in \mathbb{R}$ (in these cases $d_{J}^{3} \omega$ vanishes); $d_{J}^{4} \omega=0$ for any other parameter value.

Thus we obtain that not only the poles, but also the equator between them in $\mathcal{J}_{e}=\mathbb{S}^{2}$ consists of distinguished almost complex structures.

6.2. Homogeneous models of type $\mathbf{I I}_{2}$. Structures of type $\mathrm{II}_{2}$ also have $\mathfrak{m}=V+\mathbb{C}$ as $\mathfrak{h}$-representation. For $\mathfrak{h}=\mathfrak{s u}(1,1)$, considered as imaginary split quaternions, we identify $V$ with the module of split quaternions $\mathbb{H}_{s}$. The set of $\mathfrak{h}$-invariant complex structures $J_{q}(v)=v \cdot q, q \in \operatorname{Im}\left(\mathbb{H}_{s}\right)$ is the two sheet hyperboloid $Z^{2}=\left\{q \in \operatorname{Im}\left(\mathbb{H}_{s}\right): q \cdot q=-1\right\}$. Thus the set of $\mathfrak{h}$-invariant (almost) complex structures on $V+\mathbb{C}$ is $\mathcal{J}_{h}=Z^{2} \times\{1\} \simeq \mathbb{D}^{2} \times \mathbb{Z}_{2}$.

Notice also that on $V$ there are (almost) product structures $I_{q}(v)=v \cdot q$ forming the set $Y^{2}=\left\{q \in \operatorname{Im}\left(\mathbb{H}_{s}\right): q \cdot q=+1\right\}$, which is the one sheet hyperboloid homeomorphic to the cylinder $\mathbb{S}^{1} \times \mathbb{R}^{1}$.

$\mathbf{I I}_{2}^{a}$. The Lie group $S U(2,1)$ acts transitively on the unit pseudo-sphere in $\mathbb{C}^{3} \simeq \mathbb{R}^{4+2}$ with the metric of the signature $(4,2): Q^{5}=\left\{\left(z_{1}, z_{2}, z_{3}\right) \in \mathbb{C}^{3}\right.$ : $\left.\left|z_{1}\right|^{2}+\left|z_{2}\right|^{2}-\left|z_{3}\right|^{2}=1\right\} \simeq \mathbb{S}^{3} \times \mathbb{C}$. Thus

$$
M=U(2,1) / S U(1,1)=U(1) \times S U(2,1) / S U(1,1) \simeq \mathbb{S}^{1} \times \mathbb{S}^{3} \times \mathbb{C} .
$$

To see the invariant complex structure, notice that $\mathbb{S}^{1}$ acts on $Q^{5}, w \mapsto e^{i t} w$, and the quotient is

$$
Q^{5} / \mathbb{S}^{1}=\mathbb{C}^{2} \# \overline{\mathbb{C} P^{2}} \simeq \mathbb{C} P^{2} \backslash\{p t\} \simeq \mathbb{C} P^{1} \times \mathbb{C} ;
$$

the structure $J$ is obtained similarly to case 1 above.

Namely, the CR-connection $H \subset T Q$ carries the space $\mathcal{J}_{h}$ of $G$-invariant almost complex structures $J^{\prime}$, which form a trivial 2D bundle over $Q$. Complementing this with any shift invariant complex structure $J^{\prime \prime}$ on the torus $\mathbb{T}^{2}$, which is the fiber of the discussed map $M^{6} \rightarrow \mathbb{C} P^{1} \times \mathbb{C}$, we get the almost complex structure $J=J^{\prime}+J^{\prime \prime}$ on $M=U(2,1) / S U(1,1)$. The moduli space of these structures is $\mathcal{J}_{+}=\mathcal{J}_{h} \times \Sigma^{2}$.

If we consider the universal cover $\hat{M}=\mathbb{R}^{1} \times \mathbb{S}^{3} \times \mathbb{C}$, then the above torus becomes the cylinder $\mathbb{R}^{1} \times \mathbb{S}^{1}$ and (since on the cylinder all complex structures are equivalent) the moduli space becomes not 4- but 2-dimesional, namely $\mathcal{J}_{h}$.

Again there are two preferred structures, corresponding to $J^{\prime}= \pm i$. Indeed, moving along the orbit $\mathbb{S}^{1}$ of $U(1)$-action the space $\mathcal{J}_{h} \simeq \mathbb{D}^{2} \times \mathbb{Z}_{2}$ rotates around its axis of symmetry through $\pm i$. Only these two corresponding structures $J$ are integrable, the others have non-degenerate Nijenhuis tensor $N_{J}$.

$\mathbf{I I}_{2}^{b}$. Finally, let us discuss

$$
M=G L(3) / S U(1,1)=G L(3) / S L(2) .
$$

It has two connected components, each being simply-connected. 
We can identify $M$ with the space $\left\{\left(v, \Pi^{2}, \omega\right)\right\}$, where $v \in \mathbb{R}^{3}$ is a nonzero vector, $\Pi^{2} \not \ngtr v$ a transversal 2-plane and $\omega \in \Lambda^{2} \Pi^{*} \backslash 0$ an area form on it. Indeed, $G L(3)$ acts transitively on this space with the stabilizer $S L(2)$. Furthermore, we have

$$
M^{6}=\mathbb{R}_{*} \times N^{5}, \text { where } N^{5}=S L(3) / S L(2),
$$

and $S L(2)$ is embedded into $S L(3)$ as the lower $2 \times 2$ block.

We identify $N^{5}=\left\{(v, p) \in \mathbb{R}^{3} \times\left(\mathbb{R}^{3}\right)^{*}:\langle v, p\rangle=1\right\}$ by choosing covector $p$ in the annihilator of $\Pi$. At $a=(v, p) \in N$ the stabilizer $S L(2)$ acts on $T_{a} N=\{(w, q):\langle w, p\rangle+\langle v, q\rangle=0\}$. This has the invariant subspace $H_{a}=$ $H_{a}^{1} \oplus H_{a}^{2}$, where $H_{a}^{1}=\{(w, 0):\langle w, p\rangle=0\}$ and $H_{a}^{2}=\{(0, q):\langle v, q\rangle=0\}$.

We have: $H_{a}^{2}=\left(H_{a}^{1}\right)^{*}$. The $S L(2)$-invariant area form $\omega$ on $H_{a}^{1}$ is obtained as $\iota_{v} \Omega$, where $\Omega$ is the volume form in $\mathbb{R}^{3}$. The invariant almost complex structure is now given by $J_{r t}(\xi)=r \xi+t \hat{\xi}$, where $\xi \in H_{a}^{1}$ and $\hat{\xi}=\iota_{\xi} \omega \in H_{a}^{2}$, $r, t \in \mathbb{R}$. Requirement $J_{r t}^{2}=-1$ implies $J_{r t}(\hat{\xi})=-\frac{1+r^{2}}{t} \xi-r \hat{\xi}$. Complementing this by requirement that $J_{r t}$ maps the complement $\{(\lambda v,-\lambda p)\} \subset T_{a} N^{5}$ to $T_{a} \mathbb{R}_{*}$ we obtain the almost complex structure on $M$ parametrized by the pair $(r, t)$ or equivalently by the space $\mathcal{J}_{h}$. Direct calculation shows that none of these structures $J_{r t}$ is integrable.

The integrability properties generalizing SKT for the structures from $\mathcal{J}_{h}$ are given by the following statement.

Proposition 4. For the compatible invariant almost symplectic form $\omega$ and the operator $d_{J}=d \circ J$ we have: $d_{J}^{2} \omega \neq 0$ always; $d_{J}^{3} \omega \neq 0$ unless $J=J_{0}= \pm i$ in the case $G=U(2,1)$ ( $p=i$ in the case A3.5) or $J=\cosh t \cdot i+\sinh t \cdot k$, $t \in \mathbb{R}$ in the case $G=G L(3)$ ( $p=j$ in the case A3.5; in terms of the parameters of the Table this means $\left.q=\alpha i+\beta k, \alpha^{2}-\beta^{2}=1\right)$ - only in these cases $d_{J}^{3} \omega$ vanishes; and $d_{J}^{4} \omega=0$ for any other parameter value.

Notice that for $H=S U(1,1)$ there are less invariant structures $(J, \omega)$ with $d_{J}^{3} \omega=0$ than for $H=S U(2)$. Actually, for $G=U(2,1)$ the additional plane $\left\{q \in \operatorname{Im}\left(\mathbb{H}_{s}\right): q \perp i\right\}=\langle j, k\rangle$ consists of the product structures $q^{2}=1$; for $G=G L(3)$ and $p=j$ (we refer to the case A3.5 in Table A3) the plane $\left\{q \in \operatorname{Im}\left(\mathbb{H}_{s}\right): q \perp j\right\}=\langle i, k\rangle$ contains the indicated two-component curve of complex structures $q^{2}=-1$.

\section{Concluding Remarks}

In this paper we classified special homogeneous almost complex structures in dimension 6. Most of them turn out to be the left-invariant structures on Lie groups and, with some notable exceptions, most of these are solvable. Passing to quotient nil- or solv-manifolds destroys the isotropy, so our classification easily implies

Theorem 8. The only compact homogeneous almost complex manifolds in dimension 6 with semi-simple (nontrivial) isotropy are $\mathbb{S}^{6}, \mathbb{S}^{1} \times \mathbb{S}^{5}$ and $\mathbb{S}^{3} \times \mathbb{S}^{3}$ 
and their finite quotients, equipped with the almost complex structures as described in Theorem 1 .

Remark 5. The possible quotients, that carry general parameter almost complex structures J from Theorem 1 , are $\mathbb{S}^{1} \times\left(\mathbb{S}^{5} / \mathbb{Z}_{3}\right)$, $\mathbb{S}^{1} \tilde{\times}\left(\mathbb{S}^{5} / \mathbb{Z}_{3}\right)=$ $\left(\mathbb{S}^{1} \times \mathbb{S}^{5}\right) / \mathbb{Z}_{3}, \quad \mathbb{R P}^{3} \times \mathbb{S}^{3}$ and $\mathbb{R P}^{3} \times \mathbb{R P}^{3}$. For some exceptional values of parameters there are further quotients, e.g. the Calabi-Eckmann structure descends onto the direct product $\mathbb{S}^{1} \times\left(\mathbb{S}^{5} / \mathbb{Z}_{n}\right)$ for any $n$.

Some items from our list easily generalize to higher dimensions, providing examples of non-integrable almost complex structures (with non-degenerate Nijenhuis tensor $N_{J}$ ) with abundant symmetries. Previously some invariant almost complex structure appeared in [Wo, WG]. Here are some new examples.

For every Lie algebra $\mathfrak{h}$ and a complex vector space $(V, i)$ consider the space $\mathfrak{m}=\mathfrak{h} \otimes V$ with the $\mathfrak{h}$-invariant almost complex structure given by $J(h \otimes v)=h \otimes(i \cdot v)$. Fixing a commutative associative bilinear multiplication $Q: S^{2} V \rightarrow V$ we define the bracket by the formula

$$
\left[h \otimes v, h^{\prime} \otimes v^{\prime}\right]=\left[h, h^{\prime}\right]_{\mathfrak{h}} \otimes Q\left(v, v^{\prime}\right) .
$$

With this $\mathfrak{m}$ and $\mathfrak{g}=\mathfrak{h} \ltimes \mathfrak{m}$ become Lie algebras, and the corresponding Lie group $M=G / H$ carries $G$-invariant almost complex structure $J$.

All 4 structures in Table A2 for $\mathfrak{h}=\mathfrak{s u}(2)$ resp. Table A4 for $\mathfrak{h}=\mathfrak{s u}(1,1)$ are of this type with $V=\mathbb{R}^{2}$ (more precisely it corresponds to a semi-group structure on the set of two points). This family of generalized gradings contains, in particular, the 2-step graded nilpotent algebra $\mathfrak{h}_{1} \oplus \mathfrak{h}_{2},\left[h_{1}, h_{1}^{\prime}\right]=\left[h, h^{\prime}\right]_{2}$ (and other brackets vanish). The corresponding Lie group $M=\exp (\mathfrak{m})$ carries the left-invariant structure $J$ with non-degenerate Nijenhuis tensor in the sense $N_{J}\left(\Lambda^{2} T M\right)=T M$.

Another example is the "right extension" $M^{4 n+2}$ of the Heisenberg group over the quaternion space $\mathbb{H}^{n}$. As usual we describe the corresponding Lie algebra. Let $I, J, K$ be the triple of $\operatorname{Sp}(n)$-invariant complex structures on $\mathbb{H}^{n}$, and $\omega_{q}(x, y)=g(x q, y)$ be the symplectic structure parametrized by $q \in \mathbb{S}^{2} \subset \mathbb{R}^{3}(I, J, K)$, where $g(x, y)=x^{t} \cdot \bar{y}$ is the standard Hermitian structure, $x, y \in \mathbb{H}^{n}$. Then with fixed e $\in \mathbb{C}$ we have the following brackets on $\mathfrak{m}(\varepsilon \in\{0,1\})$ :

$$
[x, y]=\omega_{I}(x, y) \mathrm{e}, \quad[x, i \mathrm{e}]=x\left(\frac{1}{2} \varepsilon+\beta I\right), \quad[\mathrm{e}, i \mathrm{e}]=\varepsilon \mathrm{e} .
$$

Notice that $\mathbb{H}^{n} \oplus\langle\mathrm{e}\rangle$ is the Heisenberg algebra and $\mathfrak{m}$ its extension by derivations. The almost complex structure $J_{q}(x, \mathrm{e})=(x q, i \mathrm{e})$ on $M$ is invariant with respect to the Lie group $G=\operatorname{Sp}(n) \ltimes \exp (\mathfrak{m})$. The "twistor space" of this $M$ (= the bundle of moduli of the invariant almost complex structures) is the $4(n+1)$-dimensional manifold $M^{\dagger}=M \times \mathbb{S}^{2}$ equipped with the canonical almost complex structure

$$
J_{\dagger}(x, \mathrm{e}, \xi)=(x q, i \mathrm{e}, \xi q), \quad \xi \in T_{q} \mathbb{S}^{2} .
$$


This structure $J_{\dagger}$ is non-integrable (its Nijenhuis tensor is encoded by $N_{J}$ ), but has some natural pseudoholomorphic foliations.

Finally let us notice that for type II spaces from Theorem 1 the set of invariant almost complex structures $\mathcal{J}$ is two-dimensional (if we consider simply connected model $M)$ : it is parametrized by $\mathbb{S}^{2}$ for $\mathfrak{h}=\mathfrak{s u}(2)$ and by $\mathbb{Z}_{2} \times \mathbb{D}^{2}$ for $\mathfrak{h}=\mathfrak{s u}(1,1)$. The space of all these structures - the 8-dimensional "twistor space" $M^{6} \times \mathcal{J}$ - has a natural almost complex structure, but its Nijenhuis tensor is nonzero. For type $\mathrm{II}_{1}$ spaces on $M=U(3) / S U(2)$ or $M=U(2,1) / S U(2)$ (where the space of almost complex structures is given by 4 parameters) the corresponding "twistor space" $M^{6} \times \mathcal{J} \times \Sigma^{2}$ is 10 dimensional and it also possesses the natural non-integrable almost complex structure.

\section{Appendix A. Tables of the structure of $(\mathfrak{g}, \mathfrak{h}, \mathfrak{m}, J, \omega)$}

Here we list the data for Theorems 1, 3 and indicate some integrability properties for the structures. These completely encode all non-flat homogeneous almost complex manifolds with semi-simple isotropy, only excluding the special cases $G_{2}^{c} / S U(3)=S^{6}$ and $G_{2}^{*} / S U(2,1)=S^{2,4}$.

Imaginary quaternions $\mathbb{H}$ are generated by $i, j, k=i j$, which anti-commute and satisfy $i^{2}=-1, j^{2}=-1$ (and hence $k^{2}=-1$ ). In tables A1, A2 we identify $\mathfrak{h}=\operatorname{Im}(\mathbb{H})$.

Imaginary split quaternions $\mathbb{H}_{s}$ are generated by $i, j, k=i j$, which anticommute and satisfy $i^{2}=-1, j^{2}=1$ (and hence $k^{2}=1$ ). In tables A3, A4 we identify $\mathfrak{h}=\operatorname{Im}\left(\mathbb{H}_{s}\right)$.

The brackets $[\mathfrak{h}, \mathfrak{h}]$ and $[\mathfrak{h}, \mathfrak{m}]$ are straightforward and are not included into the tables. We include only the non-trivial brackets $[\mathfrak{m}, \mathfrak{m}]$.

The almost complex structure $J$ is indicated in terms of its 2 parameters for tables A1-A4 (beware that the parameter $r$ has different meaning in different tables). $J$ has no parameters in tables A5-A6. In the case of representation $\mathfrak{a d}^{\mathbb{C}}$ we use formula (11) for $J$.

We list only necessary values of the Nijenhuis tensor (to minimize the tables), the others can be restored by the rule $N_{J}(J x, y)=N_{J}(x, J y)=$ $-J N_{J}(x, y)$; we omit the trivial entries.

We write NDG to indicate that the tensor $N_{J}$ is non-degenerate; $\mathrm{DG}_{2}$ indicates that the image of $N_{J}: \Lambda_{\mathbb{C}}^{2} T M \rightarrow T M$ is a real rank 2 subdistribution of $T M, \mathrm{DG}_{1}$ means it is a real rank 4 subdistribution (in $\mathrm{DG}_{k}$ the number $k$ is the complex codimension of $\operatorname{Im}\left(N_{J}\right)$, see $\left[\mathrm{K}_{1}\right]$ ).

The Hermitian metric is given by $g(\xi, \eta)=\omega(\xi, J \eta)$ as in Section 5. Thus we describe only the almost symplectic form $\omega$ and its differential.

Recall that $V$ stands for the standard representation, $\mathbb{C}$ for the trivial complex and $\mathfrak{a} \mathfrak{d}$ for the adjoint representation. 
Table A1: isotropy $\mathfrak{h}=\mathfrak{s u}(2)$, representation $V+\mathbb{C}$ :

\begin{tabular}{|c|c|c|c|}
\hline & $\mathfrak{m}$ as $\mathfrak{h}$-representation & \multicolumn{2}{|c|}{$\mathfrak{m}=V \oplus \mathbb{C}=\mathbb{H} \oplus \mathbb{C} . \mathfrak{h}$ acts from the left, $\mathfrak{a d}_{\operatorname{Im} \mathbb{H}} \subset \operatorname{End}_{\mathbb{R}}(\mathbb{H})$} \\
\hline & vectors \& parameters & \multicolumn{2}{|c|}{$\mathrm{e} \in \mathbb{C}$ is fixed. $x, y \in \mathbb{H}$ are arbitrary. $q, p, b \in \operatorname{Im}(\mathbb{H}), \alpha, \beta, r, \varepsilon \in \mathbb{R}$} \\
\hline & structure $J$ on $\mathfrak{m}$ & \multicolumn{2}{|c|}{$J=(q, i), q^{2}=-1 . q$ acts from the right: $J(x, \mathrm{e})=(x q, i \mathrm{e})$} \\
\hline & structure $\omega$ on $\mathfrak{m}$ & \multicolumn{2}{|c|}{$\omega=\omega_{\mathbb{H}}+\omega_{\mathbb{C}}, \quad \omega_{\mathbb{H}}(x, y)=\operatorname{Re}(x b \bar{y}), \quad \omega_{\mathbb{C}}(\mathrm{e}, i \mathrm{e})=1(b \neq 0)$} \\
\hline & compatibility of $J$ and $\omega$ & \multicolumn{2}{|l|}{$b \in \mathbb{R} q$} \\
\hline \multirow[b]{2}{*}{ A1.1 } & Lie algebra structure of $\mathfrak{g}$ & tensor $N_{J} \&$ condition for $d \omega=0$ & notes \\
\hline & $\begin{array}{l}{[x, y]=\alpha \operatorname{Re}(x i \bar{y}) \mathrm{e}} \\
{[x, i \mathrm{e}]=x\left(\frac{\varepsilon}{2}+r i\right)} \\
{[\mathrm{e}, i \mathrm{e}]=\varepsilon \mathrm{e}}\end{array}$ & $\begin{array}{l}N_{J}(x, y)=\alpha(\operatorname{Re}(x q[q, i] \bar{y})-i \operatorname{Re}(x[q, i] \bar{y})) \mathrm{e} \\
N_{J}(x, \mathrm{e})=r x[q, i] \\
d \omega=0 \text { iff } \varepsilon=1, b=\alpha i\end{array}$ & $\begin{array}{l}\varepsilon \in\{0,1\}, \alpha \neq 0 \\
\text { NDG unless } r=0 \\
\text { or } q= \pm i\end{array}$ \\
\hline A 1.2 & $\begin{array}{l}{[x, y]=} \\
\quad=(\operatorname{Re}(x i \bar{y})+i \operatorname{Re}(x p \bar{y})) \mathrm{e}\end{array}$ & $\begin{array}{l}N_{J}(x, y)=\operatorname{Re}(x(q[q, i]+[q, p]) \bar{y}) \mathrm{e}+ \\
\quad+\operatorname{Re}(x(q[q, p]-[q, i]) \bar{y}) i \mathrm{e}, \quad d \omega \neq 0\end{array}$ & $\begin{array}{l}\mathrm{DG}_{2} \text { unless } \\
\qquad[p, q]=q[q, i]\end{array}$ \\
\hline A 1.3 & $\begin{array}{l}{[x, \mathrm{e}]=\alpha x} \\
{[x, i \mathrm{e}]=x(\beta+r i)} \\
{[\mathrm{e}, i \mathrm{e}]=\varepsilon \mathrm{e}}\end{array}$ & $\begin{array}{l}N_{J}(x, \mathrm{e})=r x[q, i] \\
d \omega=0 \text { iff } \alpha=\beta=0, b \in \mathbb{R} i\end{array}$ & $\begin{aligned} &(\varepsilon, \alpha) \in\{(0,0), \\
&(0,1),(1,0)\} \\
& \mathrm{DG}_{1} \text { or } \mathrm{NDG}\end{aligned}$ \\
\hline A 1.4 & $\begin{array}{l}{[x, y]=\operatorname{Re}(x i \bar{y}) \mathrm{e}+\varepsilon \cdot \mathfrak{a} \mathfrak{d}_{\operatorname{Im}(x \bar{y})}} \\
{[x, \mathrm{e}]=3 \varepsilon x i}\end{array}$ & $\begin{array}{l}N_{J}(x, y)=(\operatorname{Re}(x q[q, i] \bar{y})-i \operatorname{Re}(x[q, i] \bar{y})) \mathrm{e} \\
N_{J}(x, \mathrm{e})=3 \varepsilon x q[q, i] \\
d \omega \neq 0\end{array}$ & $\begin{array}{c}\varepsilon= \pm 1 \\
\varepsilon=-1 \Rightarrow \mathfrak{g}=\mathfrak{u}(3) \\
\varepsilon=1 \Rightarrow \mathfrak{g}=\mathfrak{u}(2,1) \\
\text { NDG unless } q= \pm i\end{array}$ \\
\hline
\end{tabular}

Table A2: isotropy $\mathfrak{h}=\mathfrak{s u}(2)$, representation $\mathfrak{a d}^{\mathbb{C}}$ :

A2.1

\begin{tabular}{|l|l|l|}
\hline $\mathfrak{m}$ as $\mathfrak{h}$-representation & $\mathfrak{m}=\mathfrak{a} \mathfrak{d} \oplus \mathfrak{a} \mathfrak{d}=\mathfrak{a} \mathfrak{d}^{\mathbb{C}}$, the complex adjoint representation of $\mathfrak{h}$ \\
\hline vectors \& parameters & $x, y \in \mathfrak{m}$. If $\mathfrak{m}=\mathfrak{m}_{a} \oplus \mathfrak{m}_{b}$, we decompose $x=x_{a}+x_{b} . r, t \in \mathbb{R}$ \\
\hline structure $J$ on $\mathfrak{m}$ & $J(u, 0)=(r u, t u), u \in \mathfrak{d} \mathfrak{d}$ and $J^{2}=-1$ \\
\hline structure $\omega$ on $\mathfrak{m}$ & $\begin{array}{l}\text { Let } K_{1}, K_{2} \text { be the Killing forms of } \mathfrak{s u}(2) \text { on each copy of } \mathfrak{a d} \text { and } \\
J_{0}(u, v)=(-v, u), u, v \in \mathfrak{a} \mathfrak{d} . \text { Then } \omega(x, y)=\left(K_{1}+K_{2}\right)\left(J_{0} x, y\right)\end{array}$ \\
\hline compatibility of $J$ and $\omega$ & $\omega$ is always compatible, never closed & notes \\
\hline \hline Lie algebra structure of $\mathfrak{g}$ & tensor $N_{J} \&$ condition for $d \omega=0$ & $\begin{array}{l}1,2 \text { are not gradings, } \\
\text { NDG always }\end{array}$ \\
\hline $\begin{array}{l}\mathfrak{m}=\mathfrak{s u}(2)_{1} \oplus \mathfrak{s u}(2)_{2} \\
\text { semi-simple }\end{array}$ & $N_{J}\left(x_{1}, y_{1}\right)=-\left(r^{2}+1\right)[x, y]_{1}+t(t-2 r)[x, y]_{2}$ & $\begin{array}{l}N_{J}=0 \text { for } J= \pm i, \\
\text { NDG else }\end{array}$ \\
\hline $\begin{array}{l}\mathfrak{m}=\mathfrak{s} \mathfrak{l}_{2}(\mathbb{C})-\operatorname{simple}, \\
\mathfrak{s u}(2) \oplus i \mathfrak{s u}(2)\end{array}$ & $N_{J}(x, y)=\left(1+r^{2}-t^{2}+2 r t i\right)[x, y]$ & $\begin{array}{l}\text { Graded } \\
\text { NDG always }\end{array}$ \\
\hline $\begin{array}{l}\mathfrak{m}=\mathfrak{s u}(2)_{0} \oplus \mathfrak{s u}(2)_{1} \\
\mathfrak{s u}(2)_{1} \text { abelian. }\end{array}$ & $N_{J}\left(x_{0}, y_{0}\right)=-\left(r^{2}+1\right)[x, y]_{0}-2 r t[x, y]_{1}$ & $\begin{array}{l}\text { Graded } \\
\text { NDG always }\end{array}$ \\
\hline $\begin{array}{l}\mathfrak{m}=\mathfrak{s u}(2)_{1} \oplus \mathfrak{s u}(2)_{2} \\
2 \text {-step nilpotent }\end{array}$ & $N_{J}\left(x_{1}, y_{1}\right)=\frac{2\left(r^{3}+r\right)}{t}[x, y]_{1}+\left(3 r^{2}-1\right)[x, y]_{2}$ & \\
\hline
\end{tabular}


Table A3: isotropy $\mathfrak{h}=\mathfrak{s u}(1,1)$, representation $V+\mathbb{C}$ :

\begin{tabular}{|c|c|c|c|}
\hline & $\mathfrak{m}$ as $\mathfrak{h}$-representation & \multicolumn{2}{|c|}{$\mathfrak{m}=V \oplus \mathbb{C}=\mathbb{H}_{s} \oplus \mathbb{C} . \mathfrak{h}$ acts from the left, $\mathfrak{a d}_{\operatorname{Im}_{\mathbb{s}}} \subset \operatorname{End}_{\mathbb{R}}\left(\mathbb{H}_{s}\right)$} \\
\hline & vectors \& parameters & \multicolumn{2}{|c|}{ e $\in \mathbb{C}$ - fixed. $x, y \in \mathbb{H}_{s}$ - arbitrary. $q, p, u, b \in \operatorname{Im}\left(\mathbb{H}_{s}\right), \alpha, \beta, r, \varepsilon \in \mathbb{R}$} \\
\hline & structure $J$ on $\mathfrak{m}$ & \multicolumn{2}{|c|}{$J=(q, i), q^{2}=-1 . q$ acts from the right: $J(x, \mathrm{e})=(x q, i \mathrm{e})$} \\
\hline & structure $\omega$ on $\mathfrak{m}$ & \multicolumn{2}{|c|}{$\omega=\omega_{\mathbb{H}_{s}}+\omega_{\mathbb{C}}, \omega_{\mathbb{H}_{s}}(x, y)=\operatorname{Re}(x b \bar{y}), \omega_{\mathbb{C}}(\mathrm{e}, i \mathrm{e})=1(b \neq 0)$} \\
\hline & compatibility of $J$ and $\omega$ & \multicolumn{2}{|c|}{$b \in \mathbb{R} q$} \\
\hline & Lie algebra structure of $\mathfrak{g}$ & tensor $N_{J} \&$ condition for $d \omega=0$ & notes \\
\hline A3.1 & $\begin{array}{l}{[x, y]=\operatorname{Re}(x p \bar{y}) \mathrm{e}} \\
{[x, i \mathrm{e}]=x\left(\frac{\varepsilon}{2}+r p\right)} \\
{[\mathrm{e}, i \mathrm{e}]=\varepsilon \mathrm{e}}\end{array}$ & $\begin{array}{l}N_{J}(x, y)=(\operatorname{Re}(x q[q, p] \bar{y})-i \operatorname{Re}(x[q, p] \bar{y})) \mathrm{e} \\
N_{J}(x, \mathrm{e})=r x[q, p] \\
d \omega=0 \text { iff } \varepsilon=1,[p, b]=p-b\end{array}$ & $\begin{array}{l}\varepsilon \in\{1,0\}, p^{2} \neq 0 \\
\text { NDG unless } r=0 \\
\text { or } q \in \mathbb{R} p\end{array}$ \\
\hline $\mathrm{A} 3.2$ & $\begin{array}{l}{[x, y]=\operatorname{Re}(x p \bar{y}) \mathrm{e}} \\
{[x, \mathrm{e}]=r x p} \\
{[x, i \mathrm{e}]=x\left(\varepsilon+\alpha \delta_{r}^{0}+u\right)} \\
{[\mathrm{e}, i \mathrm{e}]=\varepsilon \mathrm{e}}\end{array}$ & $\begin{array}{l}N_{J}(x, y)=(\operatorname{Re}(x q[q, p] \bar{y})-i \operatorname{Re}(x[q, p] \bar{y})) \mathrm{e} \\
N_{J}(x, \mathrm{e})=x(r q[q, p]+[q, u]) \\
d \omega=0 \text { iff }[u, b]=p-2\left(\varepsilon+s \delta_{r}^{0}\right) b\end{array}$ & $\begin{array}{l}\varepsilon \in\{1,0\}, p^{2}=0 \\
{[p, u]=\left(\varepsilon+2 \alpha \delta_{r}^{0}\right) p} \\
p \neq 0 ; \text { NDG unless } \\
\quad[u, q]=r q[q, p]\end{array}$ \\
\hline A 3.3 & {$[x, y]=(\operatorname{Re}(x p \bar{y})+i \operatorname{Re}(x u \bar{y})) \mathrm{e}$} & \begin{tabular}{l|l} 
& $N_{J}(x, y)=\operatorname{Re}(x(q[q, p]+[q, u]) \bar{y}) \mathrm{e}+$ \\
& $+\operatorname{Re}(x(q[q, u]-[q, p]) \bar{y}) i \mathrm{e}, \quad d \omega \neq 0$
\end{tabular} & $\begin{array}{c}\mathrm{DG}_{2} \text { if }[u, q] \neq q[q, p] \\
N_{J}=0 \text { else }\end{array}$ \\
\hline 3.4 & $\begin{array}{l}{[x, \mathrm{e}]=x(\alpha+p)} \\
{[x, i \mathrm{e}]=x(\beta+u)} \\
{[\mathrm{e}, i \mathrm{e}]=\varepsilon \mathrm{e}}\end{array}$ & $\begin{array}{l}N_{J}(x, \mathrm{e})=x(q[q, p]+[q, u]) \\
d \omega=0 \text { iff }[b, p]=[b, u]=0, \alpha=\beta=0\end{array}$ & $\begin{array}{l}\varepsilon=p=0 \\
\text { or } \varepsilon=1,[p, u]=p \\
\quad p^{2}=0, \alpha=0 \\
\text { DG }_{1} \text { or } N_{J}=0\end{array}$ \\
\hline 10.0 & $\begin{aligned} {[x, y]=} & \operatorname{Re}(x p \bar{y}) \mathrm{e}+\mathfrak{a d}_{\operatorname{Im}(x \bar{y})} \\
{[x, \mathrm{e}]=} & 3 \epsilon x p \\
& p^{2}=-\epsilon= \pm 1\end{aligned}$ & $\begin{array}{l}N_{J}(x, y)=(\operatorname{Re}(x q[q, p] \bar{y})-i \operatorname{Re}(x[q, p] \bar{y})) \mathrm{e} \\
N_{J}(x, \mathrm{e})=3 \operatorname{\varepsilon xq}[q, p] \\
d \omega \neq 0\end{array}$ & $\begin{array}{l}\quad p \in\{i, j\} \\
p=i \Rightarrow \mathfrak{g}=\mathfrak{u}(2,1) \\
p=j \Rightarrow \mathfrak{g}=\mathfrak{g l}_{3} \\
\text { NDG unless } p= \pm q\end{array}$ \\
\hline
\end{tabular}

Table A4: isotropy $\mathfrak{h}=\mathfrak{s u}(1,1)$, representation $\mathfrak{a d}^{\mathbb{C}}$ :

\begin{tabular}{|c|c|c|c|}
\hline & $\mathfrak{m}$ as $\mathfrak{h}$-representation & \multicolumn{2}{|c|}{$\mathfrak{m}=\mathfrak{a} \mathfrak{d} \oplus \mathfrak{a} \mathfrak{d}=\mathfrak{a} \mathfrak{d}^{\mathbb{C}}$, the complex adjoint representation of $\mathfrak{h}$} \\
\hline & structure $J$ on $\mathfrak{m}$ & \multicolumn{2}{|l|}{$J(u, 0)=(r u, t u), u \in \mathfrak{a} \mathfrak{d}$ and $J^{2}=-1$} \\
\hline & structure $\omega$ on $\mathfrak{m}$ & \multicolumn{2}{|c|}{$\begin{array}{l}\text { Let } K_{1}, K_{2} \text { be the Killing forms of } \mathfrak{s u}(1,1) \text { on each copy of } \mathfrak{a} \mathfrak{d} \text { and } \\
J_{0}(u, v)=(-v, u), u, v \in \mathfrak{a} \mathfrak{d} \text {. Then } \omega(x, y)=\left(K_{1}+K_{2}\right)\left(J_{0} x, y\right)\end{array}$} \\
\hline & compatibility of $J$ and $\omega$ & \multicolumn{2}{|c|}{$\omega$ is always compatible, never closed } \\
\hline & Lie algebra structure of $\mathfrak{g}$ & tensor $N_{J} \&$ condition for $d \omega=0$ & notes \\
\hline A4.1 & $\begin{array}{l}\mathfrak{m}=\mathfrak{s u}(1,1)_{1} \oplus \mathfrak{s u}(1,1)_{2} \\
\text { semi-simple }\end{array}$ & $N_{J}\left(x_{1}, y_{1}\right)=-\left(r^{2}+1\right)[x, y]_{1}+t(t-2 r)[x, y]_{2}$ & $\begin{array}{l}1,2 \text { are not gradings, } \\
\text { NDG always }\end{array}$ \\
\hline A 4.2 & $\begin{array}{l}\mathfrak{m}=\mathfrak{s l}_{2}(\mathbb{C})-\text { simple } \\
\mathfrak{s u}(1,1) \oplus i \mathfrak{s u}(1,1)\end{array}$ & $N_{J}(x, y)=\left(1+r^{2}-t^{2}+2 r t i\right)[x, y]$ & $\begin{array}{l}N_{J}=0 \text { for } J= \pm i \\
\text { NDG else }\end{array}$ \\
\hline A 4.3 & $\begin{array}{l}\mathfrak{m}=\mathfrak{s u}(1,1)_{0} \oplus \mathfrak{s u}(1,1)_{1} \\
\mathfrak{s u}(1,1)_{1} \text { Abelian }\end{array}$ & $N_{J}\left(x_{0}, y_{0}\right)=-\left(r^{2}+1\right)[x, y]_{0}-2 r t[x, y]_{1}$ & $\begin{array}{l}\text { Graded } \\
\text { NDG always }\end{array}$ \\
\hline A 4.4 & $\begin{array}{l}\mathfrak{m}=\mathfrak{s u}(1,1)_{1} \oplus \mathfrak{s u}(1,1)_{2} \\
\text { 2-step nilpotent }\end{array}$ & $N_{J}\left(x_{1}, y_{1}\right)=\frac{2\left(r^{3}+r\right)}{t}[x, y]_{1}+\left(3 r^{2}-1\right)[x, y]_{2}$ & $\begin{array}{l}\text { Graded } \\
\text { NDG always }\end{array}$ \\
\hline
\end{tabular}


Table A5: isotropy $\mathfrak{h}=\mathfrak{s l}_{2}(\mathbb{C})$, representation $V+\mathbb{C}$ :

\begin{tabular}{|c|c|c|}
\hline $\mathfrak{m}$ as $\mathfrak{h}$-representation & \multicolumn{2}{|l|}{$\mathfrak{m}=V \oplus \mathbb{C} . V \simeq \mathbb{C}^{2}$} \\
\hline vectors \& parameters & \multicolumn{2}{|c|}{ e $\in \mathbb{C}$ is fixed. $x, y \in V$ are arbitrary. $\quad \alpha, \beta, \gamma, r, \varepsilon \in \mathbb{R}$} \\
\hline structure $J$ on $\mathfrak{m}$ & \multicolumn{2}{|c|}{$J=i$ on both submodules $V$ and $\mathbb{C}$. } \\
\hline structure $\omega$ on $\mathfrak{m}$ & \multicolumn{2}{|c|}{$\begin{array}{l}\text { Let } \omega_{0} \text { be the } \mathfrak{s l}_{2}(\mathbb{C}) \text {-invariant form } 2 \text {-on } V \text { with } \mathbb{C} \text {-values. } \\
\text { Then } \omega=\omega_{V}+\omega_{\mathbb{C}}, \omega_{V}=\operatorname{Re}\left(\lambda \omega_{0}\right), \lambda \in \mathbb{C}, \omega_{\mathbb{C}}(\mathrm{e}, i \mathrm{e})=1\end{array}$} \\
\hline compatibility of $J$ and $\omega$ & \multicolumn{2}{|c|}{$\omega$ is never compatible and never closed. } \\
\hline Lie algebra structure of $\mathfrak{g}$ & tensor $N_{J} \&$ condition for $d \omega=0$ & notes \\
\hline $\begin{array}{l}{[x, \mathrm{e}]=\alpha x} \\
{[x, i \mathrm{e}]=(\beta+\gamma i) x} \\
{[\mathrm{e}, i \mathrm{e}]=\varepsilon \mathrm{e}}\end{array}$ & $N_{J}=0$ & $\begin{array}{l}(\varepsilon, \alpha) \in\{(0,0) \\
\quad(0,1),(1,0)\} \\
J \text { is integrable }\end{array}$ \\
\hline$[x, y]=(\operatorname{Re}+r \operatorname{Im}) \omega_{0}(x, y) \mathrm{e}$ & $N_{J}(x, y)=2(1-r) i \overline{\omega_{0}(x, y)} \mathrm{e}$ & $\mathrm{DG}_{2}$ unless $r=1$ \\
\hline
\end{tabular}

Table A6: isotropy $\mathfrak{h}=\mathfrak{s l}_{2}(\mathbb{C})$, representation $\mathfrak{a d}$ :

The only possible structure is $\mathfrak{m}=\mathfrak{s l}_{2}(\mathbb{C})$ and $J$ is integrable, see $\$ 5$,

There are no $\mathfrak{h}$-invariant nonzero 2 -forms on $\mathfrak{m}$.

\section{REFERENCES}

[AFS] B. Alexandrov, Th. Friedrich, N. Schoemann, Almost Hermitian 6-manifolds revisited, J. Geom.Phys. 53, 1-30 (2005).

[BKW] W. M. Boothby, S. Kobayashi, H.C. Wang, "A note on mappings and automorphisms of almost complex manifolds", Ann. Math. 77, 329-334 (1963).

[B] A. Borel, Linear algebraic groups, Graduate Texts in Mathematics 126, SpringerVerlag (1991).

[Br] R. Bryant, On the geometry of almost complex 6-manifolds, Asian J. Math. 10 (2006), no. 3, 561-605.

[Bu] J.-B. Butruille, Classification des variétés approximativement kähleriennes homogènes, Ann. Global Anal. Geom. 27, no. 3, 201-225 (2005).

[CE] E. Calabi, B. Eckmann, A class of compact, complex manifolds wich are not algebraic, Ann. of Math. 58, 494-500 (1953).

[C] É. Cartan, La théorie des groupes finis et continus et l'analysis situs, Mém. Soc. Math. 42, Paris: Gauthier-Villars (1930).

[E] C. Ehresmann, Sur les variétés presque-complexes, Proceedings of the International Congress of Mathematicians 1950, II, Amer. Math. Society, 412-419 (1952).

[F] D. B. Fuks, Cohomology of infinite-dimensional Lie algebras, Contemporary Soviet Mathematics. Consultants Bureau, New York (1986).

[G] A. Gray, Nearly Kähler manifolds, J. Differential Geometry 4, 283-309 (1970).

[GH] A. Gray, L. M. Hervella, The sixteen classes of almost Hermitian manifolds and their linear invariants, Ann. Mat. Pura Appl. (4) 123, 35 Ö58 (1980).

[GOV] V.V. Gorbatsevich, A. L. Onishchik, E. B. Vinberg, Structure of Lie groups and Lie algebras, in: Lie groups and Lie algebras III, VINITI (Russian), 41 (1990); Engl: Encyclopaedia Math. Sci. 41 Springer-Verlag (1994). 
[Ka] I. Kath, $G_{2(2)}^{*}$-structures on pseudo-Riemannian manifolds, J. Geom. Phys. 27, no. 3-4, 155-177 (1998).

[Ko] S. Kobayashi, Hyperbolic complex spaces, Springer-Verlag (1998).

$\left[\mathrm{K}_{1}\right]$ B. Kruglikov, Non-existence of higher-dimensional pseudoholomorphic submanifolds, Manuscripta Mathematica, 111 (2003), 51-69.

$\left[\mathrm{K}_{2}\right]$ B. Kruglikov, Symmetries of almost complex structures and pseudoholomorphic foliations, arXiv:1103.4404 (2011).

[KO] B. Kruglikov, M. Overholt, "The Kobayashi pseudodistance on almost complex manifolds", Diff. Geom. Appl., 11, 265-277 (1999).

[Na] P.-A. Nagy, Nearly Kähler geometry and Riemannian foliations, Asian J. Math. 6, no. 3, 481-504 (2002).

[NW] A. Nijenhuis, W. Woolf, Some integration problems in almost-complex and complex manifolds, Ann. Math. 77, 424-489 (1963).

[P] R. Palais, A global formulation of the Lie theory of transformation groups, Mem. Amer. Math. Soc. 22 (1957).

[S] N. Schoemann, Almost Hermitian structures with parallel torsion, J. Geom. Phys. 57, 2187-2212 (2007).

[V] M. Verbitsky, An intrinsic volume functional on almost complex 6-manifolds and nearly Kähler geometry, Pacific J. Math. 235, no. 2, 323-344 (2008).

[VO] E. B. Vinberg, A. L. Onishchik, Foundations of Lie Theory, VINITI (Russian), 20 (1988); Engl: Encyclopaedia Math. Sci. 20 Springer-Verlag (1993).

[Wi] H. Winther, Almost complex homogeneous spaces with semi-simple isotropy, Master thesis, University of Troms $\varnothing$ (2012).

[Wo] J. A. Wolf, The geometry and structure of isotropy irreducible homogeneous spaces, Acta Math. 120, 59-148 (1968); Correction: Acta Math. 152, 141-142 (1984).

[WG] J. A. Wolf, A. Gray, Homogeneous spaces defined by Lie group automorphisms. I, II, J. Differential Geometry 2, 77-114, 115-159 (1968).

- Boris Kruglikov, Henrik Winther: Institute of Mathematics and Statistics, University of Troms $\varnothing$, Troms $\varnothing$ 90-37, Norway.

E-mails: BORIS.KRUGLIKOV@UIT.NO, HENRIK.WINTHER@UIT.NO.

- Dmitri Alekseevsky: Masaryk University, Kotlarska 2, 61137 Brno, Czech Republic; Institute for Information Transmission Problems, B. Karetnuj per. 19, 127994, Moscow, Russia. E-mail: DALEKSEE@STAFFMAIL.ED.AC.UK 